\title{
The electron-proton bottleneck of photosynthetic oxygen evolution
}

Holger Dau ( $\nabla$ holger.dau@fu-berlin.de )

Freie Universität Berlin https://orcid.org/0000-0001-6482-7494

Paul Greife

Freie Universität Berlin

Matthias Schönborn

Freie Universität Berlin

Matteo Capone

Univ. of L'Aquila

Ricardo Assuncao

Freie Universität Berlin

Daniele Narzi

Univ. of L'Aquila

Leonardo Guidoni

Univ. of L'Aquila

\section{Biological Sciences - Article}

Keywords:

Posted Date: February 3rd, 2022

DOI: https://doi.org/10.21203/rs.3.rs-1210297/v1

License: (c) (1) This work is licensed under a Creative Commons Attribution 4.0 International License.

Read Full License 


\title{
The electron-proton bottleneck of photosynthetic oxygen evolution
}

\author{
Paul Greife ${ }^{1 \#}$, Matthias Schönborn ${ }^{1 \#}$, Matteo Capone ${ }^{2 \#, ~ R i c a r d o ~ A s s u n c ̧ a ̃ o ~}{ }^{1}$, Daniele Narzi², \\ Leonardo Guidoni ${ }^{2 *}$, Holger Dau ${ }^{1 *}$ \\ ${ }^{1}$ Dept. of Physics, Freie Univ. Berlin, Germany; ${ }^{2}$ Dept. of Physical and Chemical Sciences, \\ Univ. of L'Aquila, Italy \\ "These three authors contributed equally. \\ *Corresponding authors: holger.dau@fu-berlin.de, leonardo.guidoni@univaq.it
}

Photosynthesis fuels life on Earth by storing solar energy in chemical form, inspiring technological schemes for sustainable fuel production. Today's oxygen-rich atmosphere results from photosynthetic $\mathrm{O}_{2}$-production during water-splitting at the protein-bound manganese cluster of photosystem II. Formation of the $\mathrm{O}_{2}$ molecule starts from a state with four accumulated electron holes, the $\mathrm{S}_{4}$-state, postulated half a century ago ${ }^{1}$ and remaining enigmatic ever since. Here we resolve this missing key element in photosynthetic $\mathrm{O}_{2}$ formation and its crucial mechanistic role. We tracked 230,000 excitation cycles of darkadapted photosystems with microsecond infrared spectroscopy. Combing these results with computational chemistry reveals that in $\mathrm{S}_{4}$ not only are four electron holes accumulated by metal ion and protein sidechain oxidation, but also a crucial proton vacancy is created through gated sidechain deprotonation. Subsequently, a reactive oxygen radical is formed in an astonishing single-electron multi-proton transfer event. This is the slowest step in photosynthetic $\mathrm{O}_{2}$-formation - despite its low energetic barrier - due to entropic slowdown. In conjunction with previous breakthroughs in experimental and computational investigations, a compelling atomistic picture of photosynthetic $\mathrm{O}_{2}$-formation emerges. Our results provide insight into a biological process that has probably operated in the same unique way for three billion years and are expected to support the knowledge-based design of artificial water-splitting systems.

In all plants, algae and cyanobacteria, sunlight drives the splitting of water molecules into energized electrons and protons, both needed for reduction of $\mathrm{CO}_{2}$ and eventually carbohydrate formation. ${ }^{2}$ In this process, molecular oxygen $\left(\mathrm{O}_{2}\right)$ is formed, which about 2.4 billion years ago started to transform the Earth's atmosphere, the so-called 'great oxygenation event'. ${ }^{3}$ In light-driven water oxidation at the oxygen-evolving complex (OEC), which is a $\mathrm{Mn}_{4} \mathrm{CaO}_{5}$-cluster bound to the proteins of photosystem II (PSII), ${ }^{2,4}$ the decisive interrelation between electron and proton transfer in the bottleneck steps of $\mathrm{O}_{2}$-formation has remained 
insufficiently understood and is addressed herein in time-resolved Fourier-transform infrared (FTIR) experiments (Fig. 1).

\section{$\mathrm{O}_{2}$-transition tracked by time-resolved spectroscopy}

For time-resolved infrared spectroscopy on PSII, we developed an FTIR step-scan experiment with automated exchange of dark-adapted PSII particles (see Methods), thereby expanding previous experiments at individual wavenumbers ${ }^{5-7}$ towards detection of complete fingerprint spectra. The sample exchange system was refilled about every 60 hours using, all in all, PSII membrane particles with about $1.5 \mathrm{~g}$ of chlorophyll prepared from $40 \mathrm{~kg}$ of fresh spinach leaves, for data collection during days and nights over a period of 7 months. We initiated the transitions between semi-stable S-states by 10 visible-light $(532 \mathrm{~nm}$ ) nanosecond-laser flashes applied to the dark-adapted photosystems (Extended Data Figure 1). Employing a specific deconvolution approach based on Kok's standard model (Fig. 1a), ${ }^{1}$ the time-dependent S-state difference spectra were obtained for each of the individual transition between the 4 semistable reaction-cycle intermediates, $\mathrm{S}_{1}, \mathrm{~S}_{2}, \mathrm{~S}_{3}$, and $\mathrm{S}_{0}$.

We focus on the oxygen-evolution transition, $\mathrm{S}_{3}-\mathrm{S}_{4}->\mathrm{S}_{0}+\mathrm{O}_{2}$, predominantly induced by the third laser flash, for which time courses at selected wavenumbers are shown in Fig. 2a. Multiexponential simulations of the time courses provided 5 time-constants describing acceptor and donor side PSII processes, including the expected time constants of $340 \mu$ s and $2.5 \mathrm{~ms}$. The $2.5 \mathrm{~ms}$ time constant $\left(t_{\mathrm{O} 2}\right)$ corresponds to the reciprocal rate constant of the ratedetermining step in $\mathrm{O}-\mathrm{O}$ bond formation and $\mathrm{O}_{2}$ release, as well-known for decades. ${ }^{8,9}$ The $340 \mu$ s time constant $\left(t_{\mathrm{H}_{+}}\right)$corresponds to an obligatory step of proton removal from the oxygen-evolving complex of PSII, as shown more recently by time-resolved detection of X-ray absorption, UV-vis, recombination fluorescence and photothermal signals. ${ }^{10-12}$ Here "obligatory" means that the $\mathrm{O}-\mathrm{O}$ bond formation chemistry can proceed only after completed proton-removal, as verified by the delayed onset of signals that trace manganese oxidation states or generally the $\mathrm{O}_{2}$-formation chemistry ${ }^{9,10,12-14}$ and also visible in the time course shown in the top panel of Fig. 2a. For systematic analysis of the 2D time-wavenumber data array obtained by the FTIR step-scan experiment, we exploited that the time constants of proton removal $\left(t_{\mathrm{H}_{+}}=340 \mu \mathrm{s}\right)$ and the electron transfer associated with $\mathrm{O}_{2}$ formation $\left(t_{\mathrm{O} 2}=2.5\right.$ $\mathrm{ms}$ ) need to be wavenumber-independent because they always reflect the same reaction (the same rate constant). The time constants thus can serve as a kinetic tag of the reaction in the time-resolved spectroscopic data. By simultaneous simulation of the time courses at 2582 wavenumbers $\left(1800 \mathrm{~cm}^{-1}\right.$ to $\left.1200 \mathrm{~cm}^{-1}\right)$ using the same set of time constants at each wavenumber, the amplitude spectra shown in Fig. $2 b-d$ were obtained, which are denoted as decay-associated spectra (DAS).

\section{Pivotal proton vacancy by sidechain deprotonation}

Conventional steady-state S-state difference spectra collected hundreds of milliseconds after the laser flash could reflect the changes directly coupled to the stable light-induced oxidation state changes of manganese ions, as often assumed so far ${ }^{15,16}$ (but see also ref. ${ }^{17}$ ). Then, the 
steady-state difference spectrum should correspond to the DAS of the $t_{02}(=2.5 \mathrm{~ms})$ component, as indeed visible in Fig. $2 \mathrm{~d}$, supporting assignment to the $\mathrm{Mn} / \mathrm{Ca}$-binding carboxylate sidechains shown in Fig. $2 \mathrm{f}$. In other spectral regions, however, we observed a distinctly different behavior, where in the $t_{02}$ amplitude spectrum, (i) positive peaks at 1700 $1750 \mathrm{~cm}^{-1}$ (Fig. 2b) and (ii) negative peaks at $1565-1605 \mathrm{~cm}^{-1}$ (Fig. 2c) are not matched by counterparts in the steady-state spectrum. These peaks are likely assignable to (i) vibrations of protonated carboxylate sidechains ( $\mathrm{C}=\mathrm{O}$ vibrations of $\mathrm{Asp} / \mathrm{Glu}-\mathrm{COOH}$ ) and (ii) deprotonated sidechains of carboxylates (symmetric O-C-O vibrations of Asp/Glu-COO-). ${ }^{18}$ The mismatch between $t_{02}$ amplitudes and the near-zero steady-state spectrum is explainable by carboxylate sidechain deprotonation early in the $\mathrm{S}_{3}->\mathrm{S}_{4}->\mathrm{S}_{0}$ transition which later is reversed by reprotonation in parallel to $\mathrm{O}_{2}$-formation, as indicated by the inverted amplitude spectra of the $t_{\mathrm{H}+}$ and $t_{02}$ components (marked by red areas). On these grounds we conclude carboxylate deprotonation coinciding with relocation of a proton towards the aqueous solvent $\left(t_{\mathrm{H}_{+}}\right)$and carboxylate re-protonation paralleling the $\mathrm{O}_{2}$-formation step ( $t_{02}$ ).

Further below, the presence of three well-resolved bands at 1707, 1723 and $1744 \mathrm{~cm}^{-1}$ (Extended Data Figure 4) is attributed to a specific pair of carboxylate residues with fluctuating $\mathrm{H}$-bond configurations, the Glu65-Glu212 pair in Fig. 1c, which may described as a proton gate ${ }^{19}$ or proton loading site. ${ }^{20}$ In analogy to the proton loading site of cytochrome c-oxidase, ${ }^{21}$ also (fractional) protonation state changes of also further groups might be involved. In the following, based on a recent crystallographic model of the OEC in its $\mathrm{S}_{3}$-state, ${ }^{22}$ we explore a plausible carboxylate assignment and identify the $\mathrm{S}_{4}$ and $\mathrm{S}_{4}$ ' states computationally.

\section{Atomistic scenario by energy-path calculations}

We investigated the reaction path leading to $\mathrm{O}-\mathrm{O}$ bond formation by DFT-based minimum energy path (MEP) calculations (see Methods). Resulting structures, energy levels and spin states along the sampled path are summarized in Fig. 3 and Extended Data Figs. 7 and 8. The computational tracing of $\mathrm{O}-\mathrm{O}$ bond formation starts in $\mathrm{S}_{4}$, where the redox-active tyrosine $\left(\mathrm{Tyr}_{\mathrm{z}}\right)$ is oxidized, but the $\mathrm{Mn}_{4} \mathrm{Ca}$ cluster still is in the $\mathrm{S}_{3}$-state conformation which previously has been determined by serial free-electron laser crystallography. ${ }^{22,23}$ Moreover, it is assumed that the transition to the $S_{4}$-state has been completed (within about $300 \mu \mathrm{s}$ ), resulting in a deprotonated state of a carboxylate group, which is the prerequisite for the subsequent reactions leading via the $\mathrm{S}_{4}$ ' state to $\mathrm{O}-\mathrm{O}$ bond formation and $\mathrm{O}_{2}$-release.

We identify the $S_{4}{ }^{\prime}$ state as a metastable state where (i) the proton previously located in a strong $\mathrm{H}$-bond between the two substrate oxygen atoms, $\mathrm{O5}$ and $\mathrm{O6}$, is gone and (ii) one electron has been transferred from $\mathrm{O} 6$ to $\mathrm{Tyr}$, resulting in an oxyl radical terminally bound to a $\mathrm{Mn}^{\mathrm{IV}}$ ion. ${ }^{24,25}$. This means that the $\mathrm{Tyr}_{\mathbf{z}}{ }^{\bullet}$ hole is transferred to 06 , which constitutes oxidation of a substrate-water oxygen by one electron. Such $\mathrm{S}_{4}$ ' state cannot be considered a transition state, but rather a reaction intermediate which is stable (minimally) over the 10 ps trajectory of the ab-initio MD simulations. ${ }^{26}$ 
The obvious mechanistic problem of the $\mathrm{S}_{4}->\mathrm{S}_{4}{ }^{\prime}$ transition is that both (i) $\mathrm{Mn}^{\mathrm{IV}}-\mathrm{OH}$ deprotonation without previous hole transfer to $\mathrm{Tyr}_{\mathrm{z}}{ }^{\mathrm{ox}}$ and (ii) hydroxyl radical formation without previous deprotonation would involve an energetically unfavorable intermediate state. Now our MEP calculations show how this mechanistic problem can be solved via the coupled (concerted) movement of one electron (to $\mathrm{Tyrz}^{\mathrm{ox}}$ ) and three protons (Figure 3, Supporting video). The results of our calculations show that the proton movement can be viewed as a Grotthus-type proton transfer: the individual protons are shifted within an $\mathrm{H}$-bond by only about $1 \AA$, whereas overall the protonation is moved over a distance of $\sim 7 \AA$, from 06 to Asp61.

The MEP calculations result in an energetic barrier of $\mathrm{Mn}^{\mathrm{IV}}-\mathrm{O}^{\bullet}$ formation in the $\mathrm{S}_{4}->\mathrm{S}_{4}{ }^{\prime}$ transition of only $7 \mathrm{kcal} / \mathrm{mol}(300 \mathrm{meV})$. This figure agrees surprisingly well with the experimental value of $7.1 \mathrm{kcal} / \mathrm{mol}(310 \mathrm{meV})$, which we determined for the crystallographically characterized cyanobacterial photosystems from Thermosynechococcus elongatus from the temperature dependence of the rate constant of $\mathrm{O}_{2}$-formation (Arrhenius plot) by applying Eyring's transition-state theory (Eyring, 1935), see Extended Data Fig. 5. Assuming a purely enthalpic free-energy of activation, these values would imply a time constant below $1 \mu \mathrm{s}$, whereas a millisecond time constant is detected. The experimental findings thus indicate a significant entropic contribution to the free energy of activation, of about $6.5 \mathrm{kcal} / \mathrm{mol}$ ( $285 \mathrm{meV}$ ), which corresponds to a slowdown by a factor exceeding 10000 . The pronounced entropic slowdown is most plausibly explained by numerous approximately isoenergetic conformations of the $\mathrm{H}$-bonded protein-water network at the active site, with rapid interconversion between these networks. Out of these conformations, a subset of specific arrangements of the atoms and $\mathrm{H}$-bond interactions is required for the ratedetermining reaction to proceed. Pronounced variations in water positions and $\mathrm{H}$-bonded chains of water molecules, coupled to protein dynamics, indeed have been observed in classical MD simulation of PSII in the time range of tens of nanoseconds, ${ }^{27}$ rendering a strong entropic contribution to any reaction likely that relies on a specific location of water molecules and $\mathrm{H}$-bond interactions, as is the case for the $\mathrm{S}_{4}->\mathrm{S}_{4}{ }^{\prime}$ transition shown in Fig. 3 . The crystallographic analysis of semi-stable intermediates in the water oxidation cycle also supports reaction steps coupled to rearrangement of water molecules. ${ }^{22,28}$

Our MEP calculations suggest protonation of the Asp61 carboxylate group in an electron transfer step that is coupled to a Grotthus-type movement of three protons, with the unconventional feature of a metal ion ( $\mathrm{Mn} 4)$ acting as a relay. This single-electron-multiproton step facilitates the critical step of $\mathrm{Mn}^{\mathrm{IV}}-\mathrm{O}^{\bullet}$ formation $\left(\mathrm{S}_{4}{ }^{\prime}\right)$ prior to O-O bond formation. The transient carboxylate deprotonation and reprotonation we detected in the IR experiment might be assignable to the sidechain of Asp61. However, Asp61 likely is deprotonated already in the $S_{3}$ steady state of the Kok cycle (refs. ${ }^{29-32}$ and section SII.1 of the Supplementary Information). In an alternative scenario, we identify a proton-carrying carboxylate 'dyad', Glu312 and Glu65, as the site of deprotonation within about $300 \mu$ s after the flash, where presumably Glu312 is initially protonated (ref. $^{33}$, section SII.2 of the Supplementary 
Information) and transiently deprotonated during the oxygen evolution transition. Other computational work suggests Glu65 as initially protonated, ${ }^{34}$ but this difference here is of minor importance only. A shift of the nearby Lys317 sidechain could play a role in promoting Glu312/Glu65 deprotonation and increasing the proton affinity of Asp61, as shown in Extended Data Fig. 9 and section SII.3 of the Supplementary Information. Noteworthily, Yano and coworkers ${ }^{21}$ suggested that the Glu65-Glu212 pair serves as a proton-transport gate, based on transient conformational changes during the $S_{2}->S_{3}$ transition detected by serial crystallography. They did not investigate the oxygen-evolution transition itself. Also, they could not prove deprotonation of the carboxylate dyad. Nonetheless, assuming transferability to the here investigated $\mathrm{S}_{3} \rightarrow \mathrm{S}_{4}->\mathrm{S}_{0}$ transition, their finding of conformational changes of Glu65 provides significant support to our conclusion of $\mathrm{Tyr}_{\mathrm{z}}$-oxidation inducing residue rearrangements and Glu65-Glu212 deprotonation.

\section{Conclusions}

We identify the rate-determining step in photosynthetic water oxidation as formation of a $\mathrm{Mn}^{\mathrm{IV}}$-O $\mathrm{O}^{\bullet}$ radical, which corresponds to a one-electron oxidation of a substrate water molecule. The close coupling between electron transfer and $\mathrm{Mn}-\mathrm{OH}$ deprotonation via Grotthus-type relocation of three protons facilitates the low enthalpic reaction barrier, with a computed barrier height that is as low as found experimentally. The concerted electron-proton reaction requires that the nuclei and $\mathrm{H}$-bond pattern at the active site are perfectly well arranged, which provides a plausible explanation of reaction slowdown by entropic factors. Computationally we find a low-energy path for the subsequent steps leading to O-O bond formation, which is in line with a mechanistic proposal reported already in 2008 by Siegbahn ${ }^{35,36}$ and recent structural data. ${ }^{22,37}$ However, we cannot exclude further low-energy routes towards $\mathrm{O}-\mathrm{O}$ bond formation, ${ }^{32}$ as they inter alia have been discussed in relation to substrate-water exchange rates ${ }^{38}$ because an exhaustive search covering all conceivable reaction paths is unachievable. We emphasize that Siegbahn and others ${ }^{32,39,40}$ did not clarify the mechanism of $\mathrm{Mn}-\mathrm{OH}$ deprotonation and $\mathrm{Mn}^{\mathrm{IV}}-\mathrm{O}^{\bullet}$ radical formation, which we identify as the kinetically most demanding, overall bottleneck step in photosynthetic water oxidation.

For starting the reactions leading to $\mathrm{O}_{2}$-formation, the formation of $\mathrm{Tyr}_{\mathrm{z}}{ }^{\mathrm{ox}}$, which provides the fourth electron hole at the active site, is insufficient; the creation of a proton vacancy by removal of a further proton from the active site is required. ${ }^{10}$ We now find that the proton is not removed from a substrate water molecule, but from a nearby base, which is identified as a carboxylate sidechain. This is the prerequisite for the subsequent concerted electron-proton transfer reaction that facilitates in the initial one-electron oxidation of a substrate water molecule. The last detectable state before onset of water oxidation thus is formed in the presence of four accumulated electron holes by creating an essential proton vacancy. We believe that its characteristics justify assignment to the pivotal $\mathrm{S}_{4}$-state, whose identity had remained enigmatic since Bessel Kok developed the S-state cycle paradigm 50 years ago. ${ }^{1}$ 
An important development in (photo)electrocatalytic water oxidation for employment in $\mathrm{CO}_{2}$ neutral fuel production is the use of oxide materials based on earth-abundant metals ( $\mathrm{Mn}, \mathrm{Fe}$, $\mathrm{Co}, \mathrm{Ni}$ ). These often share structural motifs with the biological catalyst and frequently undergo metal-centered oxidation state changes, which allows accumulation of oxidizing equivalents before onset of $\mathrm{O}_{2}$ formation, ${ }^{41-43}$ e.g. by $\mathrm{Mn}^{\text {III/IV }}$ oxidation. ${ }^{44}$ In the light of the knowledge gained for photosynthetic water oxidation, we consider it plausible that also in the inorganic oxide materials, the formation of a reactive metal- $\mathrm{O}^{\bullet}$ radical is the kinetically most demanding rate-determining step, which can proceed efficiently by coupled electron-proton transfer. Following the biological paragon, we expect that tuning the inorganic material for (i) extensive hole accumulation by metal oxidation at low overpotentials and (ii) metal-radical formation facilitated by proton-coupled electron transfer can lead to improved OER catalyst materials based on earth-abundant resources. 

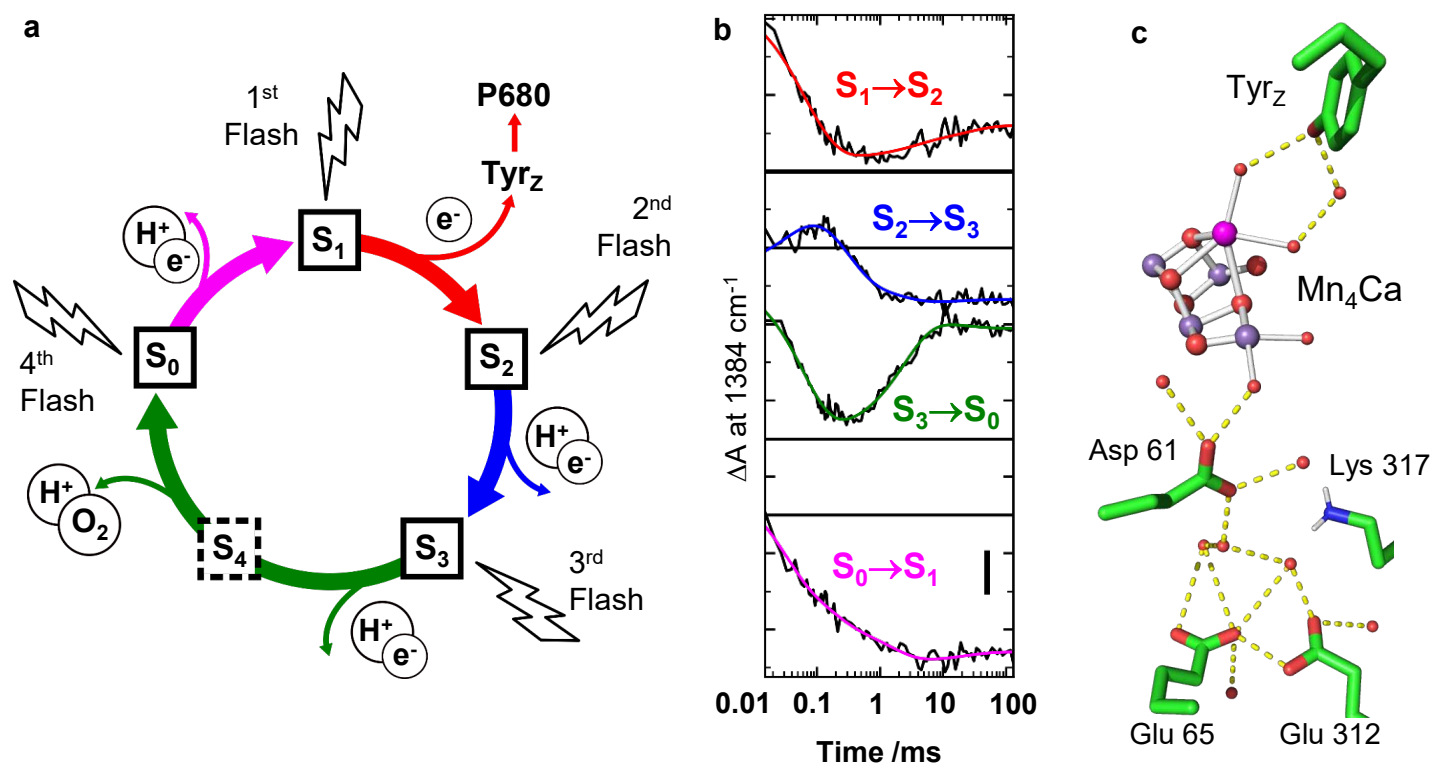

Fig. 1. Reaction cycle of photosynthetic oxygen evolution. a) Basic S-state cycle model: Starting in the dark-stable $\mathrm{S}_{1}$-state, each laser flash initiates oxidation of the primary chlorophyll donor ( $\mathrm{P} 60^{+}$ formation) followed by electron transfer from a tyrosine sidechain ( $\mathrm{Tyr}_{\mathrm{z}}$ oxidation) and, in three of the four S-state transitions, manganese oxidation until four electron holes (oxidizing equivalents) are accumulated in the $\mathrm{S}_{4}$-state. b) Example of tracing S-state transitions by infrared absorption changes (here at $1384 \mathrm{~cm}^{-1}$ ) after excitation with visible-light laser flashes. The scale bar corresponds to $25 \mu \mathrm{OD}$. c) $\mathrm{Mn}_{4} \mathrm{Ca}$ cluster ( $\mathrm{Mn}$, violet; $\mathrm{Ca}$, green) in the $\mathrm{S}_{3}$-state with six bridging oxygens, the redox-active tyrosine ( $\mathrm{Tyr}_{\mathrm{z}}$ ), and further selected protein sidechains as well as water molecules (red spheres). 


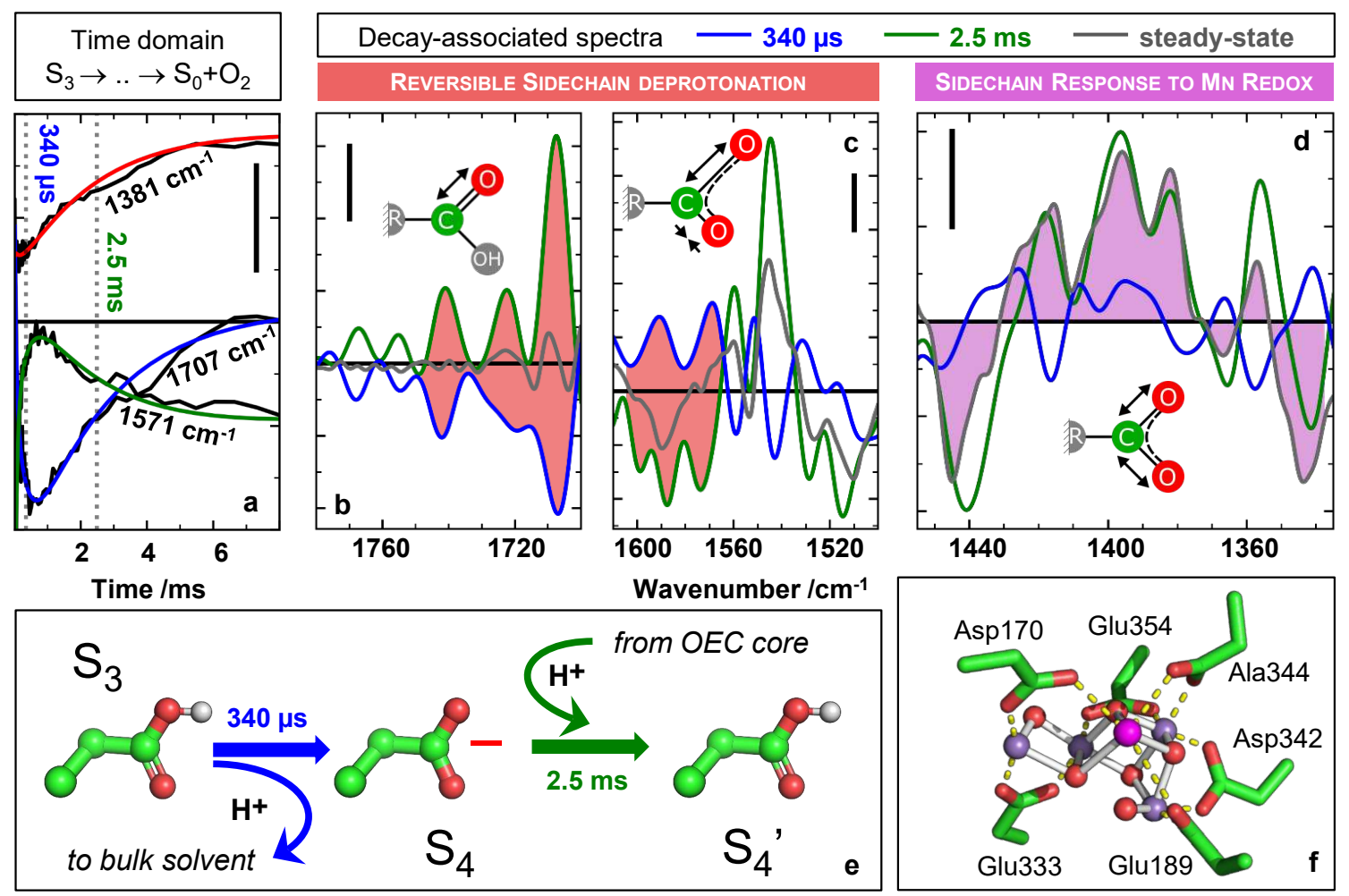

Fig. 2. Oxygen-evolution transition traced by FTIR. a) IR time traces at selected wavenumber demonstrating the delayed onset of $\mathrm{O}-\mathrm{O}$ bond formation $\left(1381 \mathrm{~cm}^{-1}\right)$ and reversible changes assignable to transient sidechain deprotonation $\left(1571 \mathrm{~cm}^{-1}\right.$ and $\left.1707 \mathrm{~cm}^{-1}\right) . \mathbf{b}, \mathbf{c}$, and d) DAS corresponding to the proton release phase $\left(t_{\mathrm{H}^{+}}=340 \mu \mathrm{s}\right.$, blue line) and the oxygen-evolution phase ( $t_{\mathrm{O} 2}=2.5 \mathrm{~ms}$, green) as well as the steady-state difference spectrum of the $S_{3} \rightarrow S_{0}+O_{2}$ transition (grey). Red areas in $b$ and $d$ mark inverted $340 \mu \mathrm{s}$ DAS and $2.5 \mathrm{~ms}$ DAS, indicating reversible behavior; purple shaded areas in c mark similarity of $2.5 \mathrm{~ms}$ DAS and steady-state spectrum, in line with assignment to non-transient $\mathrm{Mn}$ oxidation state changes. e) Transient deprotonation of carboxylate sidechains as implied by the data of $b$ and $c$. f) $\mathrm{Mn} / \mathrm{Ca}$-binding protein sidechains relating to the spectra in $\mathrm{d}$. 

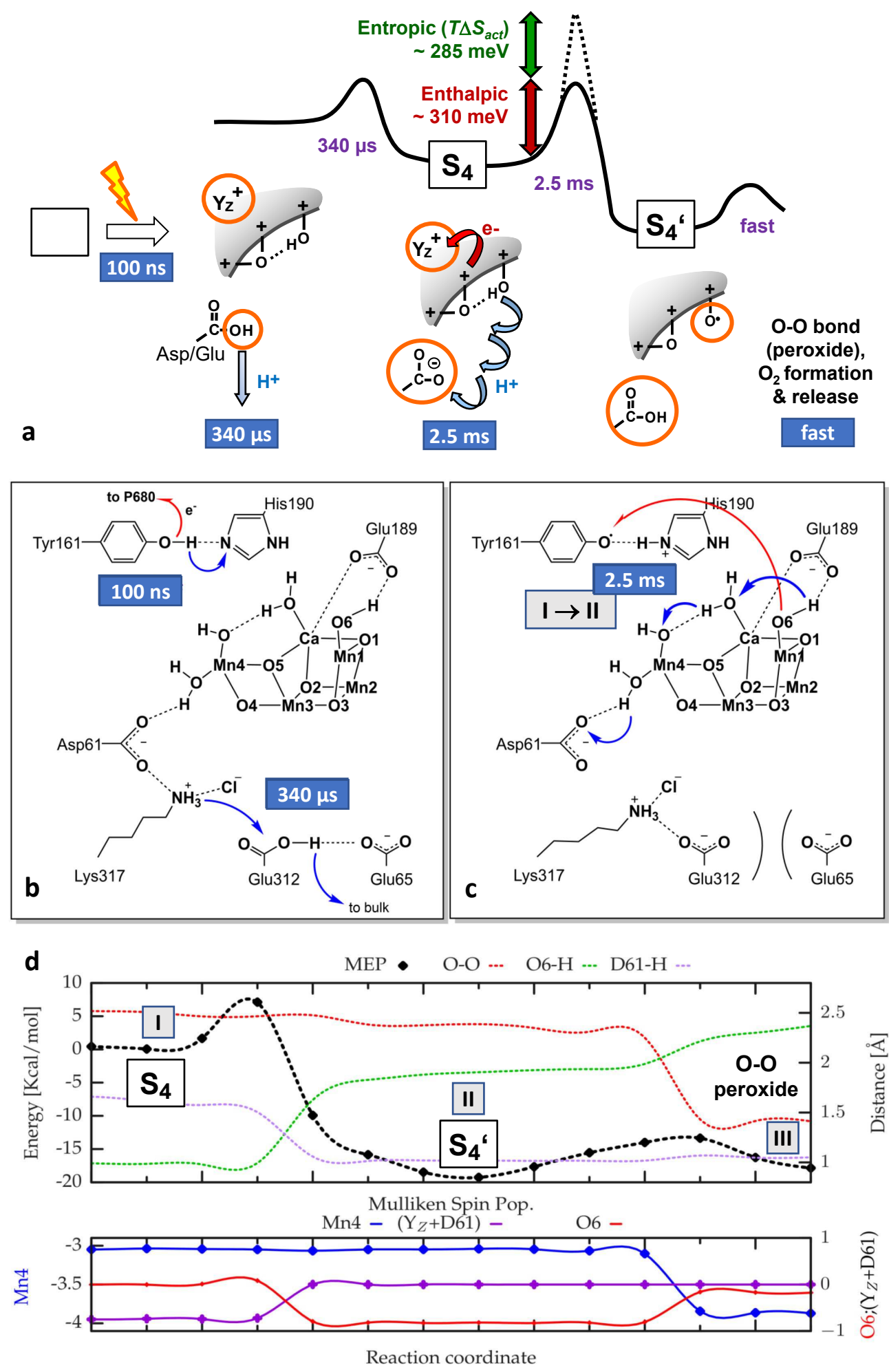

Fig. 3. Proton and electron transfer steps of the oxygen-evolution transition. In a), summary of experimental findings (Figure 2 and Extended Data Figure 5). Based on computational analysis, b) creation of proton vacancy in the $S_{3} \rightarrow S_{4}$ and $c$ ) onset of water oxidation by coupled electron-proton transfer in $\mathrm{S}_{4} \rightarrow \mathrm{S}_{4}{ }^{\prime}$. d) Energy values associated with MEP calculations and internuclear distances (dashed lines) as well as spin populations (solid lines) that characterize the peroxide formation. See Extended Data Figures 7 and 8, for structures complementing panel $d$ and describing the complete oxygen-evolution transition. 


\section{Methods}

\section{Preparation of PSII particles}

High-activity PSII membrane particles ( $>1000 \mu \mathrm{mol} \mathrm{O}_{2}$ per $\mathrm{mg} \mathrm{Chl} \mathrm{and} \mathrm{h)} \mathrm{were} \mathrm{prepared} \mathrm{from} \mathrm{spinach}$ leaves as described previously, ${ }^{45}$ with a Triton incubation time reduced to $1 \mathrm{~min},{ }^{46}$ and stored at $-80^{\circ} \mathrm{C}$ in a betaine-rich cryo-buffer (1 M glycinebetaine, $25 \mathrm{mM}$ 2-morpholin-4-ylethanesulfonic acid (MES), $15 \mathrm{mM} \mathrm{NaCl}, 5 \mathrm{mM} \mathrm{MgCl}_{2}, 5 \mathrm{mM} \mathrm{CaCl}_{2}, \mathrm{pH}$ 6.2) with a final chlorophyll (Chl) concentration of about $2 \mathrm{mg} / \mathrm{mL}$. Starting with $4 \mathrm{~kg}$ of spinach leaves, each PSII preparation yielded PSII membrane particles corresponding to about $200 \mathrm{mg} \mathrm{Chl}$. In total, about $40 \mathrm{~kg}$ of fresh spinach leaves were processed for this work, resulting in about PSII membrane particles corresponding to more than $1500 \mathrm{mg}$ of chlorophyll.

Prior to measurement, the PSII membrane particles were thawed, resuspended and washed twice by centrifugation at $50,000 \mathrm{~g}$ for 12 minutes in betaine-free variant of the cryo-buffer. The resulting pellet was mixed with an artificial electron acceptor (about $30 \mu \mathrm{L}$ of a stock solution of $700 \mathrm{mM}$ phenyl-pbenzoquinone (PPBQ) dissolved in dimethyl sulfoxide (DMSO)) yielding a final ratio of $7 \mu \mathrm{mol} P P B Q$ per $\mathrm{mg} \mathrm{Chl}$ of the PSII membrane particles.

\section{Realization of 1800 irradiation spots}

The FTIR measurement cells consisted of two $\mathrm{CaF}_{2}$ plates (diameter of $25 \mathrm{~mm}$ ) sealed with vacuum grease. A small amount of PSII suspension with added electron acceptor sample was placed on one plate, with the second plate pressed firmly on top. The thickness of the sample was set using a selfmade device using rotation and pneumatic pressure to press the plates together; simultaneous measurement of the optical density (OD) at $1650 \mathrm{~cm}^{-1}$ allowed for the reasonably homogenous samples with an OD of 1+/-0.2 OD. Following thickness adjustment, two laser flashes were applied (5 ns pulse width, $532 \mathrm{~nm}$ ). These pre-flashes and the subsequent dark-relaxation period ensure a reproducible synchronization of the photosystems in the dark-stable $S_{1}$-state with the tyrosine residue denoted as Tyr $_{\mathrm{D}}$ in its oxidized form. ${ }^{47}$

Approximately $45 \mathrm{CaF}_{2}$ 'sandwiches', prepared as described above, were loaded into an automated sample change plate and mounted within a modified commercial FTIR spectrometer (Bruker Vertex 70, detector $\mathrm{D} 317 / \mathrm{BF}$ ) with an extended sample chamber and temperature control device, as shown in Extended Data Figure 1 and detailed in section SI.1 of the Supplementary Information. Concave focusing mirrors further focused the IR beam to a spot with a diameter of about $1.6 \mathrm{~mm}$ on the PSII sample. Each of the $45 \mathrm{CaF}_{2}$ sandwiches can have about 40 unique sample spots, resulting in about 1800 individual spots per refill of the automated sample exchange system. Prior to measurement, the OD of each spot is sampled, and those not meeting the criteria of $1+/-0.2$ OD are excluded from further measurement. This typically resulted in the exclusion of about $10 \%$ of the spots.

\section{Execution of the step-scan FTIR experiment}


The temperature was regulated to $10^{\circ} \mathrm{C}$ and a constant stream of dry air kept the sample chamber humidity below $2 \%$. S-state cycling of the PSII membrane particles was achieved using saturating flashes from an excitation laser ( 5 ns flashes, $532 \mathrm{~nm}$, Continuum Minilight II) coupled into the sample chamber through a transmission window. Spot size was adjusted using an iris to be larger than the IRbeam diameter.

After loading the spectrometer and before start of the step-scan FTIR experiment, the samples were dark-adapted for minimally one hour. In the FTIR experiment, dark-adapted spots of the PSII sample were irradiated with 10 excitation laser flashes with a flash spacing of $700 \mathrm{~ms}$. For each flash, the detector signal was recorded for $136 \mathrm{~ms}$, with a 24-Bit analog-to-digital conversion every $6 \mu \mathrm{s}$ (sampling frequency of about $170 \mathrm{kHz}$ ), 6 ms before the flash and $130 \mathrm{~ms}$ after the flash, as illustrated by the timing diagram in Extended Fig. 1. Once data collection for the $10^{\text {th }}$ flash had been completed, the sample holder moved a 'fresh' sample spot into the IR beam path before initiation of the next flash sequence. At least one hour of dark adaptation passed before the same sample spot was measured again. For the step-scan data set, 334 interferogram mirror positions were repeatedly sampled, resulting in a spectral resolution of about $10 \mathrm{~cm}^{-1}$. For signal averaging, about 230,000 measurement cycles where performed, meaning about 2,300,000 excitation flashes were applied and recorded within about 7 months. During this period, the experimental set-up was repeatedly refilled with fresh PSII FTIR samples and data for total measurement time of about 60 days was recorded. After extensive assessment of the loss of functional stability over extended time periods, we decided to expose the FTIR PSII samples to laser flash sequences for typically 50-60 h (see Supplementary Figure 2). The primary data analysis as well as the correction for thermal artefacts are described in the Supplementary Information.

For comparison, rapid-scan S-state difference spectra were recorded using the same type FTIR samples and automated sample exchange system as used for the step-scan data. The individual rapid-scan interferograms were collected within $37 \mathrm{~ms}$, at a spectral resolution of $4 \mathrm{~cm}^{-1}$. The steady state was calculated by averaging spectra from $300 \mathrm{~ms}$ to $1 \mathrm{~s}$ after each laser flash.

\section{Analysis via decay-associated spectra (DAS)}

The time evolution of the obtained spectra was simulated with a standard exponential model:

$$
\left.F_{v}(t)=\sum_{i} A_{v, i} \cdot\left(1-e^{-t / \tau_{i}}\right)+B_{v}, \quad \text { (Eq. } 1\right)
$$

where $F_{v}(\mathrm{t})$ provides spectrum at a specific time, $t$, after the laser flash and $v$ indicates discrete wavenumbers spaced by $0.23 \mathrm{~cm}^{-1}$. The time constants $\left(\tau_{\mathrm{i}}\right)$ and amplitude coefficients, $A_{v, i}$, were obtained by a simultaneous least-square fit of 2582 transients corresponding to wavenumbers ranging from $1800 \mathrm{~cm}^{-1}$ to $1200 \mathrm{~cm}^{-1}$.

The amplitude coefficients, $A_{v, i}$, when plotted with respect to the wavenumber, represents the change in the recorded spectrum associated with time constant $\tau_{i}$, the decay-associated spectrum (DAS) of the $\tau_{i}$-process. Note that according to Eq. 1 , a positive value for $A_{v, i}$ represents a positive contribution the final steady-state spectrum. The offset $B_{v}$ represents the spectrum of all flash-induced changes 
that are faster than the time-resolution of our experiment (sum of changes occurring within nanosecond up to about $5 \mu$ s after the laser flash). Simulations begin $9 \mu$ s after the excitation flash.

\section{Acceptor side correction}

In the decay-associated spectrum (DAS) of the $S_{3}->S_{0}$ transition, the amplitude of the millisecond phase is a combination (sum) of a minor contribution from the quinone reactions at the PSII acceptor side and major contribution of the oxygen-evolution step. For improved DAS of the oxygen-evolution step, we corrected for the quinone contribution, based on the following rationale: The two-electron chemistry at the PSII acceptor side results in binary oscillation of the flash-number dependent acceptor side contribution, with one type of acceptor side contributions dominating on odd flash number and a second type on even flash numbers. This is supported by the clear similarity between the millisecond decay-associated spectra of the $S_{2}->S_{3}$ and $S_{0}->S_{1}$ transitions (see Supplementary Fig. 3). Consequently, we assume that the DAS of the millisecond phase in the $S_{1}->S_{2}$ transition also contributes to the millisecond DAS in the $S_{3}-S_{0}$ transition.

For the $S_{1}->S_{2}$ transition, decay-associated spectra were obtained for 4 time-constant components: 33 $\mu \mathrm{s}, 91 \mu \mathrm{s}, 3.1 \mathrm{~ms}$, and $25 \mathrm{~ms}$. The DAS of the $3.1 \mathrm{~ms}$ phase was subtracted from the corresponding DAS of the $\mathrm{S}_{3} \rightarrow \mathrm{S}_{0}$ transition, without further scaling. We note that the acceptor side correction is uncritical to our conclusion, as demonstrated in Supplementary Fig. 4, but improves the precision of the decay associated spectra shown in Fig. 2.

\section{Time-resolved $\mathrm{O}_{2}$-polarography and determination of act and $\mathrm{S}_{\text {act }}$}

Thylakoid membranes from $T$. elongatus cells were prepared as described elsewhere. ${ }^{48}$ After storage at $-80{ }^{\circ} \mathrm{C}$, the thylakoid suspension was thawed on ice, for $60 \mathrm{~min}$ in complete darkness. After resuspension in an electrolyte-buffer $\left(150 \mathrm{mM} \mathrm{NaCl}, 25 \mathrm{mM} \mathrm{MES}, 1 \mathrm{M}\right.$ glycinebetaine, $5 \mathrm{mM} \mathrm{MgCl}_{2}, 5$ $\mathrm{mM} \mathrm{CaCl}{ }^{2}, \mathrm{pH} 6.2$ adjusted with $\mathrm{NaOH}$ ), an aliquot of $10 \mu \mathrm{L}$ with a PSIl concentration corresponding to $10 \mu \mathrm{g}$ of chlorophyll was put into the cavity of a centrifugable two-electrode assembly followed by centrifugation in a swing-out rotor at 10,000 g. We used a custom-made centrifugable static ring-disk electrode assembly of a bare platinum and silver-ring electrodes to perform time-resolved oxygen polarography measurements. ${ }^{48,49} \mathrm{~A}$ custom-made potentiostat provided the polarization voltage ($0.95 \mathrm{~V}$ at the $\mathrm{Pt}$ electrode versus the $\mathrm{Ag}$ ring electrode) for $\mathrm{O}_{2}$-reduction at the bare Pt electrode, which was switched-on $15 \mathrm{~s}$ before the first excitation flash. The current signal was recorded for $500 \mathrm{~ms}$ (20 ms before and $480 \mathrm{~ms}$ after each flash, for 80 flashes with $900 \mathrm{~ms}$ spacing) using a first-order high-pass filter (time constant of $100 \mathrm{~ms}$ ) for suppression of slow drift contributions in the current signal. The Sstate transitions were induced by saturating flashes of red light ( $613 \mathrm{~nm}, 40 \mu \mathrm{s}$ of flash duration). The light-source was a high-power light-emitting diode (LED) operated at a maximum current density of ca. $150 \mathrm{~A}$, as facilitated by capacitor discharge. The temperature during data acquisition was set using Peltier elements and monitored by a miniature temperature sensor immersed in the sample buffer.

For accurate (correct) and precise determination of the $\mathrm{O}_{2}$-formation rate constant, the recorded current transients were simulated by numerical integration of the one-dimensional $\mathrm{O}_{2}$ diffusion 
equation, involving light-induced $\mathrm{O}_{2}$-production within the PSII layer covering the electrode (source terms), $\mathrm{O}_{2}$-consumption at the electrode (sink terms), and accounting for the high-pass filter characteristics (see refs. ${ }^{48,49}$, software developed by Dr. Ivelina Zaharieva). By variation of model parameters, the recorded $\mathrm{O}_{2}$-transients were simulated until optimal agreement between experimental and simulated transients was reached (least-square curve-fitting).

After determination of the activation energy $\left(E_{\text {act }}\right)$ and pre-exponential factor $(A)$ following the classical approach of Arrhenius, the enthalpy of activation $\left(H_{\text {act }}\right)$, entropy of activation $\left(S_{\text {act }}\right)$ were determined using the Eyring equation of transition-state theory (also called Eyring-Polanyi equation) with a transmission coefficient of unity, analogous to the treatment in refs. ${ }^{50,51 .}$

\section{QM/MM MD simulations}

The model used for QM/MM calculations is the same adopted in previous studies ${ }^{24,26}$. The model consists of the D1, D2 and CP43 protein domains, the respective co-factors, the $\mathrm{Mn}_{4} \mathrm{Ca}$ cluster, and water molecules present in this region. The $\mathrm{Mn}_{4} \mathrm{Ca}$ cluster, with its ligands present in the first shell (Asp170, Glu189, His332, Glu333, Asp342, Ala344 and CP43-Glu354), plus additional residues in the second shell (Asp61, Tyr161, His190, His337, Ser169 and CP43-Arg357) were treated the DFT level. Additionally, the first 14 water molecules closest or directly coordinated to the $\mathrm{Mn}_{4} \mathrm{Ca}$ cluster, and the chloride anion close to Glu333 were also treated at DFT level. The rest of the system was treated at classical level using AMBER99SB force field ${ }^{52}$ to describe the protein residues and the general AMBER force field $(\mathrm{GAFF})^{53}$ for the description of the other cofactors present in the investigated region of PSII.

$\mathrm{QM} / \mathrm{MM}$ calculations reported in this study have been carried out using the CP2K package ${ }^{54}$. QM/MM MD simulations were performed in the NVT ensemble using a Nose-Hoover thermostat ${ }^{55,56}$ (time constant $\tau=0.1 \mathrm{ps}$ ) to couple the system with a thermal bath at $\mathrm{T}=298.15 \mathrm{~K}$. A cutoff for the planewave expansion of 320 Rydberg was used to treat the quantum region with a cubic cell $28.0 \times 28.0 \mathrm{x}$ 28.0 A. The PBE+U scheme ${ }^{57}$ was employed using the DZVPMOLOPT-SR-GTH Gaussian basis set optimized for molecular systems. ${ }^{58}$ Electrostatic coupling between the classical and quantum regions of the system was treated by means of fast Gaussian expansion of the electrostatic potential. ${ }^{54} \mathrm{~A}$ time step of $0.5 \mathrm{fs}$ was used.

In order to study the effect of the protonation of Asp61 by W1 water molecule, we applied a position restraint on the proton between Asp61 and W1 to force the protonation of Asp61. The O-H equilibrium distance for the restraint was set to $1 \mathrm{~A}$ with a force constant of 0.01 internal units. The restraint has been applied for a limited amount of time and the results are described in supplementary information.

\section{Minimum Energy Path calculations}

Minimum energy path (MEP) calculations have been performed on a PSII gas-phase model directly extracted from the QM treated region of the QMMM simulations described in ref. ${ }^{24}$. The B3LYP ${ }^{59,60}$ functional with TZVP-MOLOPT-SR-GTH Gaussian basis set has been employed for all the gas-phase calculations. A $28.0 \AA$ A side cubic cell and a cut-off for the plane-wave expansion of 320 Rydberg was used to treat the quantum region. The systems have been simulated following the "high oxidation state paradigm" of the $\mathrm{S}_{3}{ }^{+}$state, consistent with previous computational work ${ }^{24,32,36,61-63}$ and in line with the 
majority of experimental analyses, as reviewed in ref. ${ }^{64}$, albeit it may not be possible to exclude the "low oxidation state paradigm" entirely. The "high oxidation state paradigm" in the $\mathrm{S}_{3}{ }^{+}$state corresponds to an oxidation pattern of the four $\mathrm{Mn}$ ions equal to IV, IV, IV, IV. The spin multiplicity corresponds to $M=\left(2 m_{s}+1\right)=6$ with a spin moment of $5 / 2$ as determined experimentally. ${ }^{65}$

As starting structures for the MEP, we used a linear interpolation between the initial and the final geometries of the selected reaction pathway. The MEP has been calculated employing the nudged elastic band (NEB) method as implemented in CP2K package ${ }^{58,66}$. The algorithm form of NEB calculation applied to our model is the improved tangent version (IT-NEB) ${ }^{67}$ on a set of 16 replicas, which include both proton reorganization and peroxide bond formation. MEP calculations have been carried out using the CP2K package ${ }^{54}$. This setup has been previously applied successfully ${ }^{61,62}$.

\section{Classical MD Simulations}

Two Classical MD simulations have been performed in order to investigate the possible conformations adopted by Lys317. The first simulation (Simulation 1) was carried out considering both Asp61 and CP43-Glu312 as deprotonated. In the second simulation (Simulation 2) Glu312 of the CP43 subunit was set as protonated, while Asp61 was still deprotonated. Apart from this difference, the two simulations are based on the same setup described in ref. ${ }^{68}$. Protein residues were described using the AMBER99SB force field ${ }^{53}$ while other molecules present in the crystal structure (i.e. $\beta$-carotene, chlorophyll a, pheophytin a, plastoquinone 9, heptyl 1-thiohexopyranoside, dodecyl- $\beta$-D-maltoside and the four lipids DGDG, MGDG, PG and SQDG) have been described by the generalized Amber force field (GAFF). ${ }^{53}$ Optimization and electrostatic potential analysis were performed by using Gaussian 03 [Gaussian03] at the Hartree-Fock level with the 6-31G* basis set. The protonation state of the histidines bound to heme molecules, or directly interacting with iron atoms or the magnesium atom in chlorophylls was chosen accordingly with their relative orientation in the X-ray structure. The other titratable residues were considered in their standard protonation state with the only exception of Glu312 of CP43 domain in Simulation 2 considered protonated. The PSII structure was embedded into a membrane bilayer composed by DOPC lipids described by the GAFF-based force field developed by Siu et al. ${ }^{69}$. The system was then solvated in a box with dimensions $27.0 \times 16.3 \times 14.2 \mathrm{~nm}$ using TIP3p water model. ${ }^{70} \mathrm{MD}$ simulations were performed using the GROMACS software package. ${ }^{71}$ Long-range electrostatic interactions were calculated using Particle Mesh Ewald (PME) method, ${ }^{72}$ with a grid spacing of $0.12 \mathrm{~nm}$ and a short-range cutoff of $1.0 \mathrm{~nm}$. The LINCS algorithm ${ }^{73}$ was applied to constrain the bond lengths of the hydrogen atoms to a constant value. A time step of $2 \mathrm{fs}$ was used for numerical integration of the equations of motion. The temperature was kept constant by coupling the system to Nose-Hoover thermostat $(298 \mathrm{~K})$ with a coupling time constant $\tau_{T}=0.1 \mathrm{ps.}{ }^{55,56}$ The system was also weakly coupled to a pressure bath (1 bar) with a coupling time constant $\tau_{\mathrm{p}}=1.0 \mathrm{ps}$, using Parrinello-Rahman barostat. ${ }^{74,75}$ The final system was composed by almost 650,000 atoms. The two MD simulations were carried out for 50 ns in NPT ensemble.

\section{Data Availability}

The data required to support our conclusions is presented in the article, in the Extended Data Figures and in the Supplementary Information file. Experimental data at intermediate stages of data analysis is available from one of the corresponding authors, $\mathrm{HD}$, upon reasonable request. 


\section{Additional Information}

\section{Acknowledgements}

The FTIR step-scan experiment on PSII was developed by Dr. Björn Süss (2008 - 2011, automated sample exchange scheme and collection of very first FTIR step-scan data on PSII) ${ }^{76}$, Dr. Mikaela Görlin (presently Uppsala University, 2011 - 2012 investigation of heat artefact contributions and collection of preliminary step-scan data) ${ }^{77}$ and Dr. Petko Chernev (presently Uppsala University, development of control software and data collection until 2018). We thank them for their basic and indispensable contributions to development of the experiment. We gratefully acknowledge Dr. Yvonne Zilliges, Dr. Z. Rebeca Pérez and Janis Hantke for skilful photobioreactor operation (for growth of T. elongatus) and preparation of the cyanobacterial thylakoid membranes. We thank Prof. Robert Burnap (Oklahoma State Univ.) and Dr. Michael Haumann (Berlin) for stimulating discussions and critical reading of the manuscript. P.G., M.S., R.A. and H.D. gratefully acknowledge financial support by the Deutsche Forschungsgemeinschaft (DFG, German Research Foundation) provided to the collaborative research center on Protonation Dynamics in Protein Function (SFB 1078, project A4/Dau) and under Germany's Excellence Strategy - EXC 2008/1 - 390540038 - UniSysCat. M.C., D.N., and L.G. gratefully thank the computational resources provided by the Partnership for the Advanced Computing in Europe (PRACE, project "Pra14_3574") and CINECA computer centre (project "IsC37_QMMMS3S4).

\section{Author contributions}

H.D. and L.G. designed the experimental and computational research, respectively. M.S. optimized the FTIR step-scan experiments and collected the data. P.G. analyzed the FTIR data. R.A. collected and analyzed the $\mathrm{O}_{2}$-polarography data. M.C. and D.N. performed the computational research. H.D., L.G. and P.G. wrote the paper.

\section{Competing interests.}

The authors declare no competing interests.

\section{Additional Information}

Supplementary information is available for this paper: (i) one Supplementary Information file providing further information on data collection, data analysis, and computational analyses; (ii) one video file illustrating the nuclear rearrangement associated with both, the $S_{4}$ to $S_{4}{ }^{\prime}$ transition and the subsequent peroxide formation step.

Correspondence should be addressed to H.D and L.G. 


\section{Extended Data Figures}

a
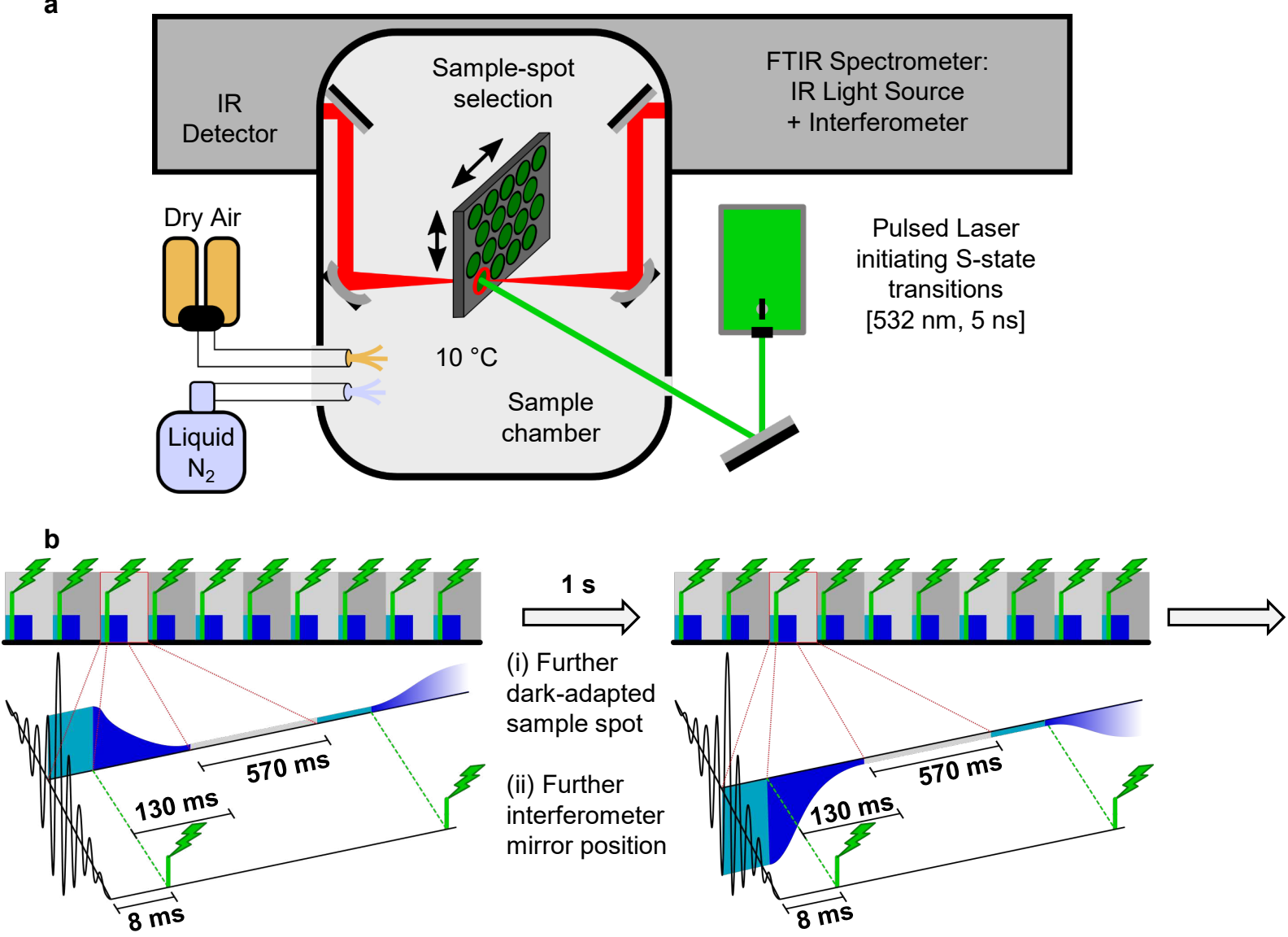

(i) Further dark-adapted sample spot

(ii) Further interferometer mirror position

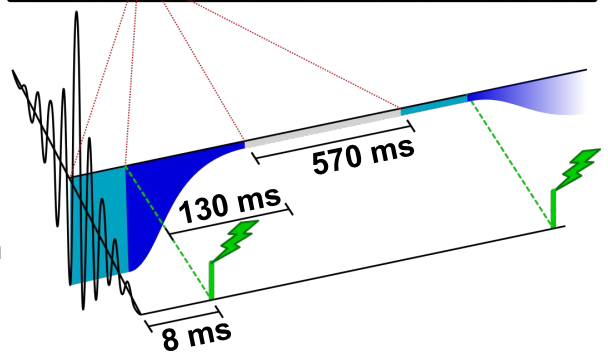

C
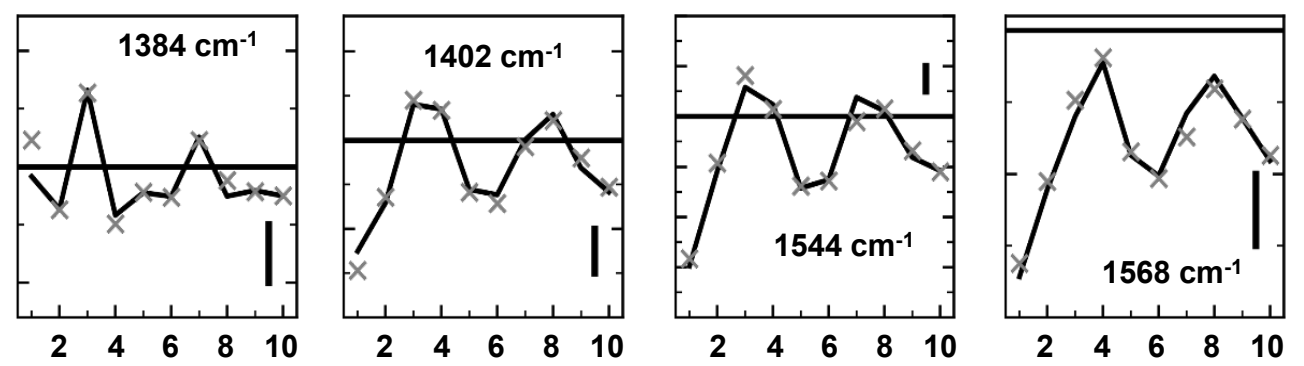

Flash Number

Extended Data Fig. 1: Experimental setup, timing scheme, and infrared data confirming synchronized reaction-cycle advancement. a. Schematic representation of the experimental setup. A standard FTIR instrument was modified with an extended, air-tight sample chamber to harbour the automated sample exchange system and combined with a pulsed nanosecond laser. After removal of water vapor from ambient air (dry air generator), the dry gas stream flooded the sample changer. Within the sample changer the temperature was kept constant by a flow of cold nitrogen gas, which had been created by occasional injection of liquid nitrogen into a Dewar vessel, regulated such that the temperature stayed close to $10^{\circ} \mathrm{C}$. Stepping motors moving the sample state in $\mathrm{x}$-y direction facilitated laser-flash excitation (and data collection) at 1800 spots of dark-adapted PSII samples. b. Timing scheme of the experiment. At each sample spot, 10 sequential flashes were applied while the 
interferometer had been set to a specific mirror position. The IR detector recorded $8 \mathrm{~ms}$ before each flash, $130 \mathrm{~ms}$ after each flash and waited $570 \mathrm{~ms}$ between the flashes without recording data. Once the detector had finished recording after the tenth flash, the sample changer moved to a 'fresh' dark-adapted sample spot and the interferometer mirror moved to a new position within about $1 \mathrm{~s}$. The 10-flash sequence was applied again at the new positions and the whole timing sequence was repeated numerous times. c. By application of 10 sequential laser flashes, the PSII can cycle up to 2.5 times through its S-state cycle. The synchronized advancement in the S-state cycle is verified by a period-offour pattern in the infrared absorption changes (here averaged from 50-130 ms after the laser flash for 4 selected wavenumbers). Solid lines show the data points and the grey cross represent a simulation using the deconvolved S-States with the derived miss factor and starting populations. Scale bars correspond to $25 \mu \mathrm{OD}$. 

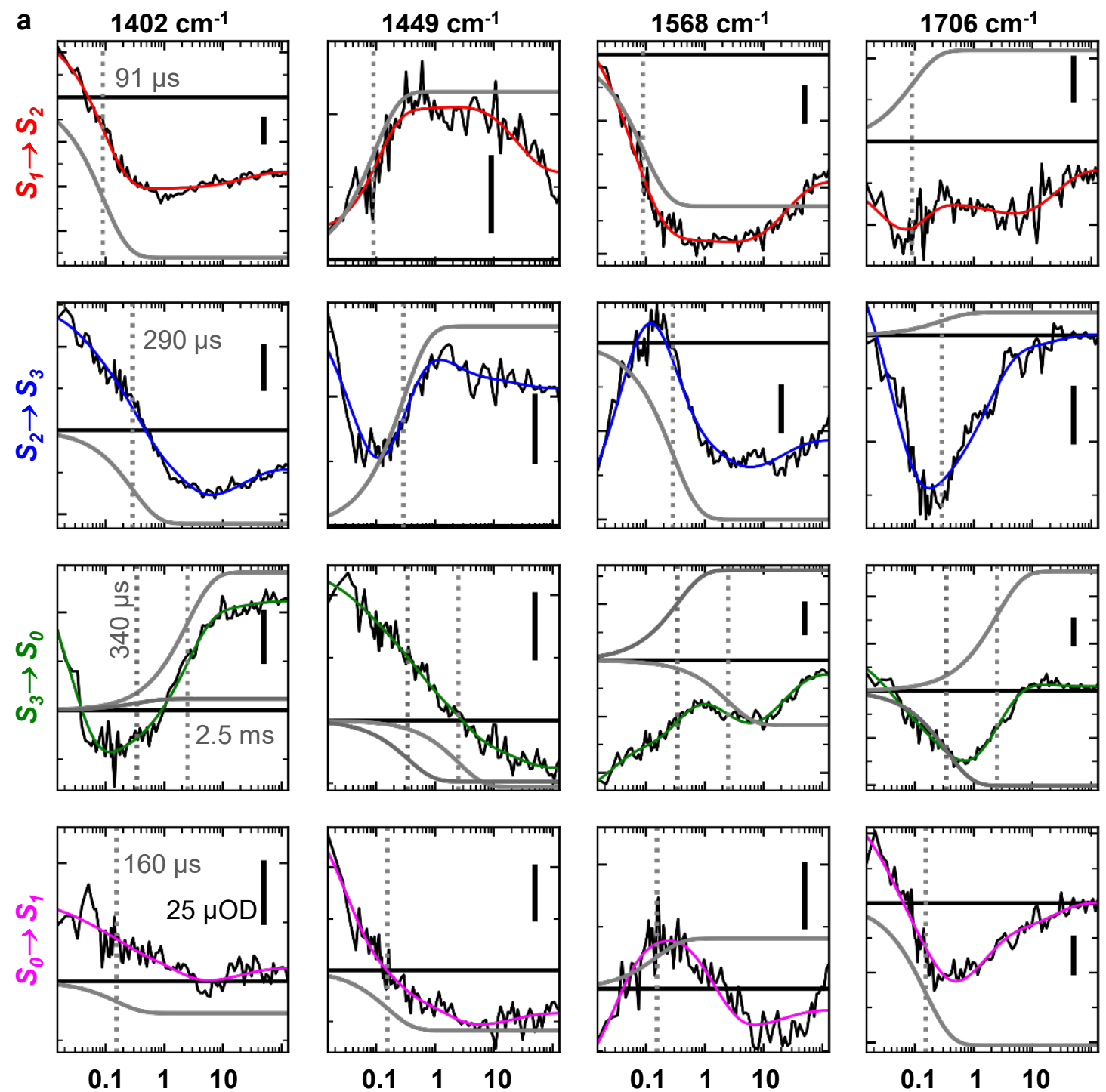

Time /ms

b

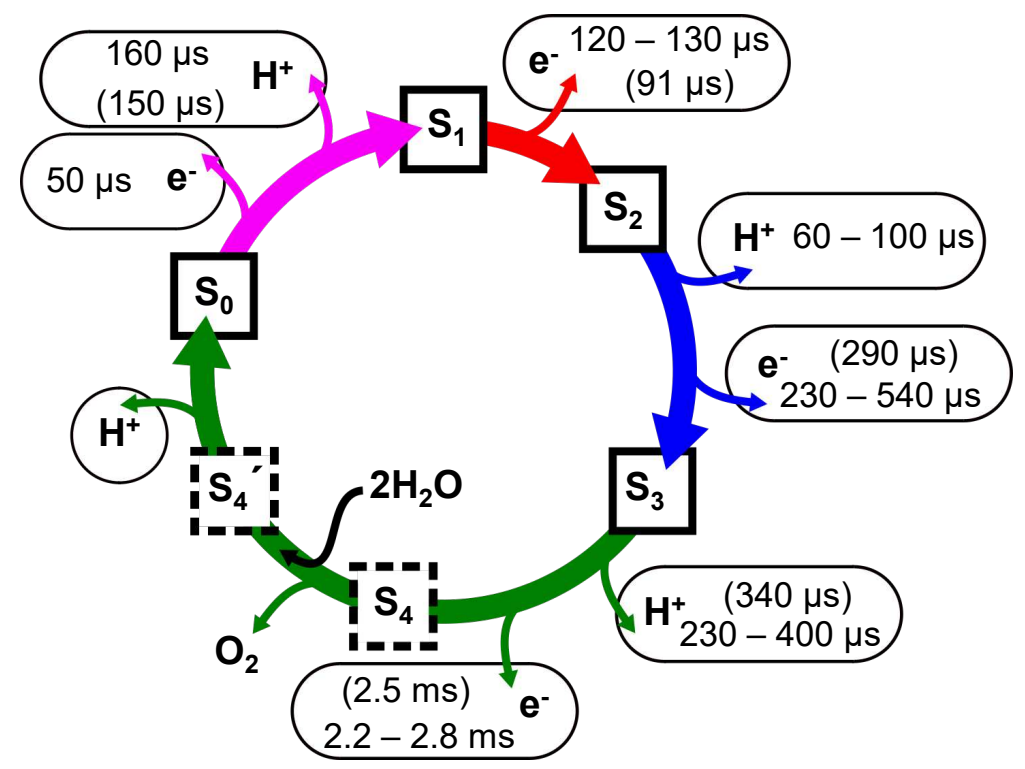


Extended Data Fig. 2: Select infrared transients demonstrating that previously identified rate constants are resolved also in the step-scan FTIR experiment. a. Transients at select wavenumbers for the four deconvolved S-state transitions. These transients are shown to demonstrate that previously identified reaction kinetics are recoverable in this work. Smooth coloured lines represent multi-exponential simulation (least-square fit) of the IR transients. Grey dashed lines mark the identified time constants while grey transients show the respective simulated contributions to the total transient. The complete set of simulation parameters can be found in Supplementary Table 1. b. S-state cycle with previously determined time constants values (see Supplementary Table 2 ) and those found in this work (in parenthesis). 


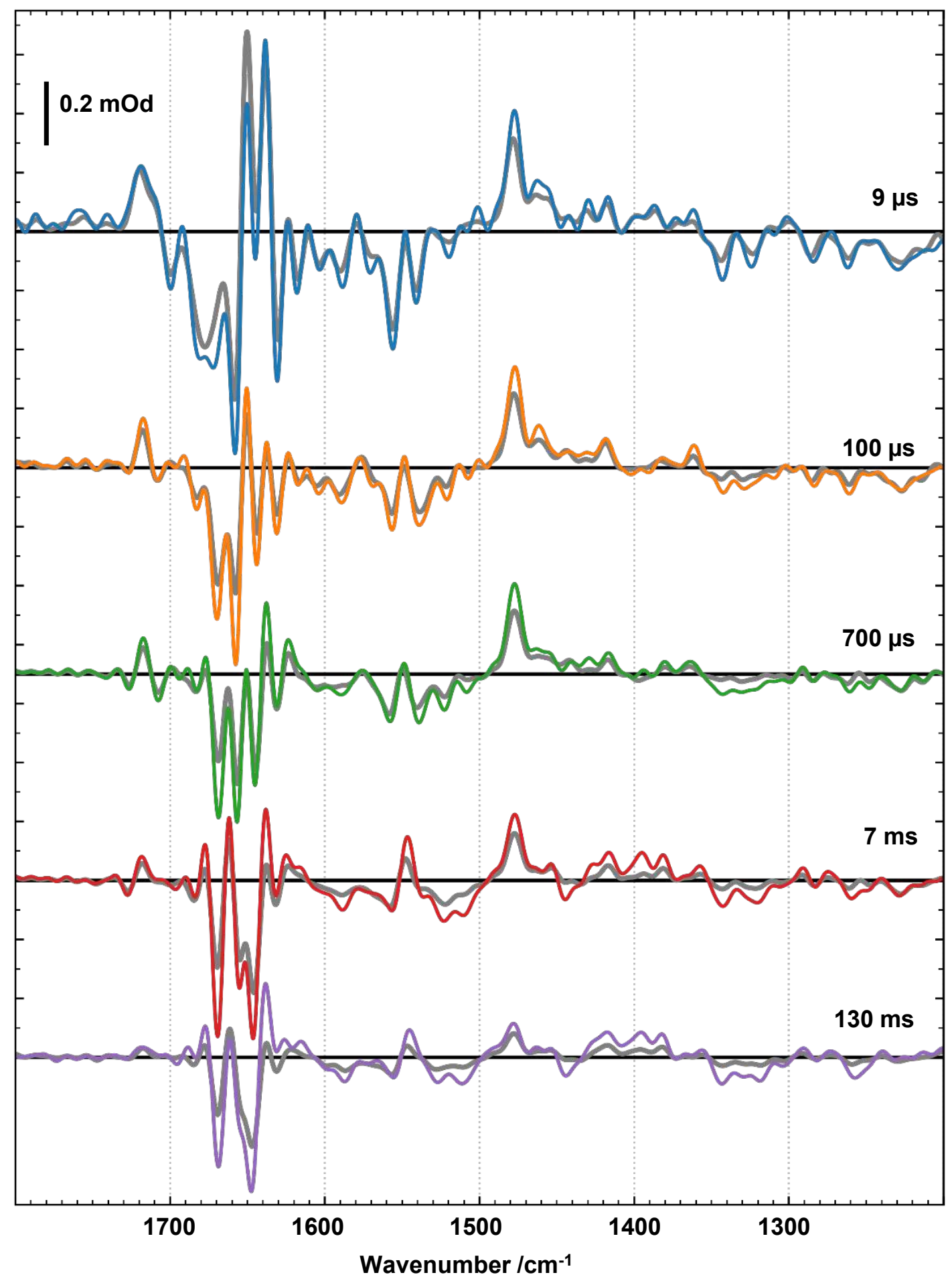

Extended Data Fig. 3: Infrared spectra at various times after application of the laser that initiates the oxygen-evolution transition. The grey line shows the spectral changes induced by the $3^{\text {rd }}$ laser flash applied to dark-adapted PSII. The coloured lines are corrected (deconvolved) for imperfect advancement in the S-state cycle as detailed in the Supplementary Material. 

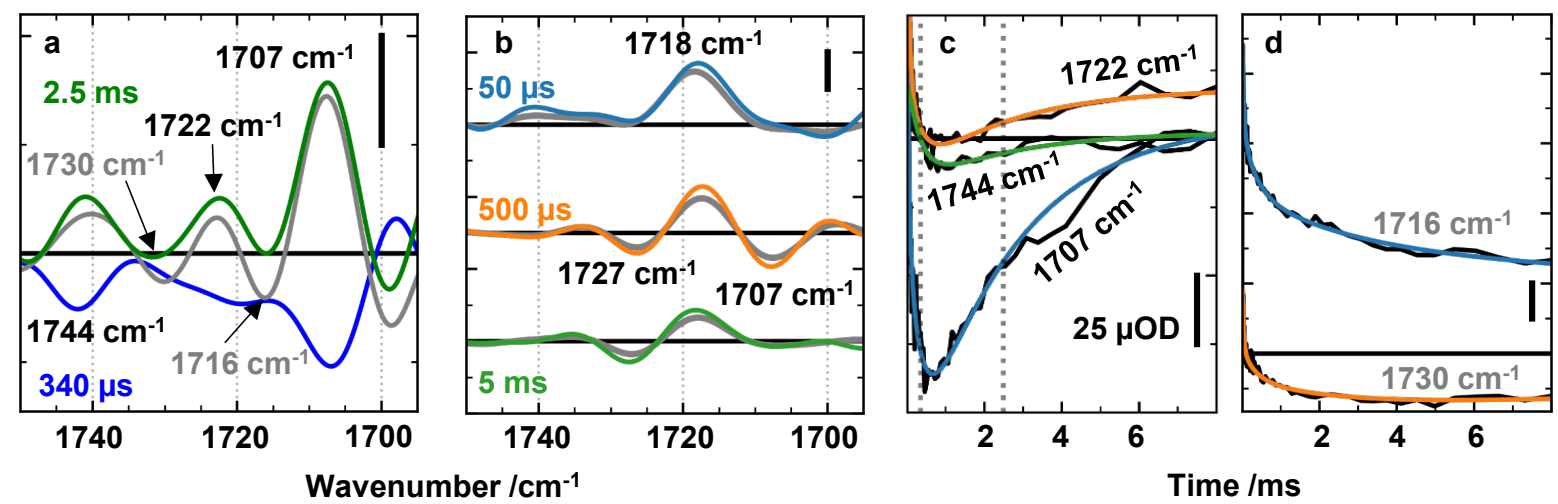

Extended Data Fig. 4: Carbonyl band spectra and IR transients between 1695 and $1750 \mathrm{~cm}^{-1}$ for the oxygen-evolution transition $\left(\mathbf{S}_{3} \rightarrow \mathbf{S}_{4}->\mathbf{S}_{0}\right)$. a. Decay-associated spectra of the $340 \mu$ s component and of the $2.5 \mathrm{~ms}$ component, the latter before (grey line) and after (green line) correction for acceptor side contributions. The acceptor side correction results in three positive peaks for the $2.5 \mathrm{~ms}$ component, indicating that band shift cannot explain these three peaks at $1730 \mathrm{~cm}^{-1}, 1722 \mathrm{~cm}^{-1}$ and $1707 \mathrm{~cm}^{-1}$. b. Spectra at selected times for the oxygen-evolution transition induced by the $3^{\text {rd }}$ laser flash before (grey lines) and after (coloured lines) deconvolution. The high level of similarity indicates that in this spectral region the deconvolution correction is uncritical as it hardly modifies the $3^{\text {rd }}$-flash data. c. Transients displaying reversible behaviour of the $340 \mu \mathrm{s}$ and $2.5 \mathrm{~ms}$ phases reflecting carboxylate deprotonation and reprotonation. d. Transient changes assignable to acceptor side contributions. All scale bars correspond to $25 \mu \mathrm{OD}$. For details on correction for acceptor side contributions and S-state deconvolution, see Supplementary Information. 

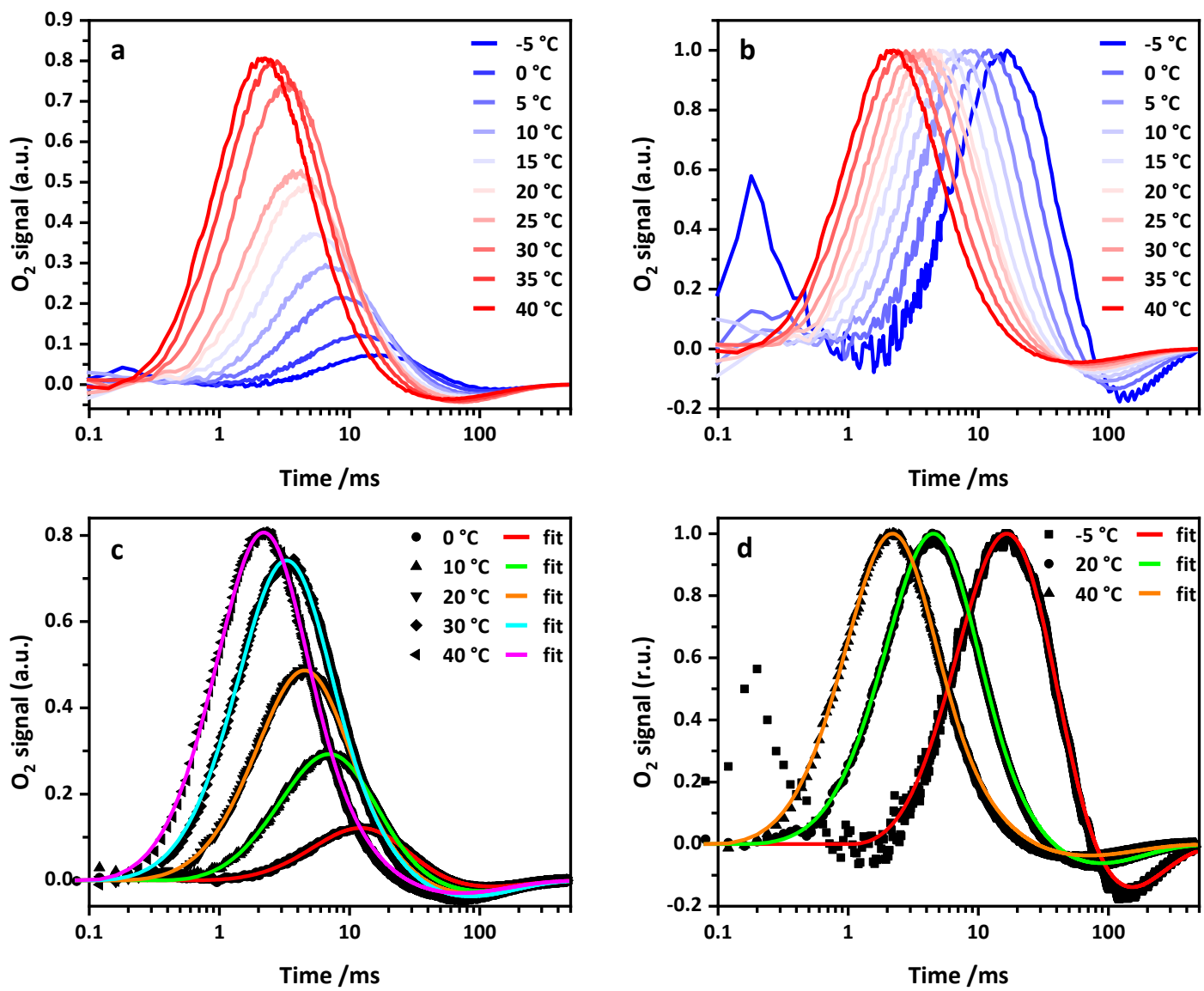

Temperature $\left({ }^{\circ} \mathrm{C}\right)$

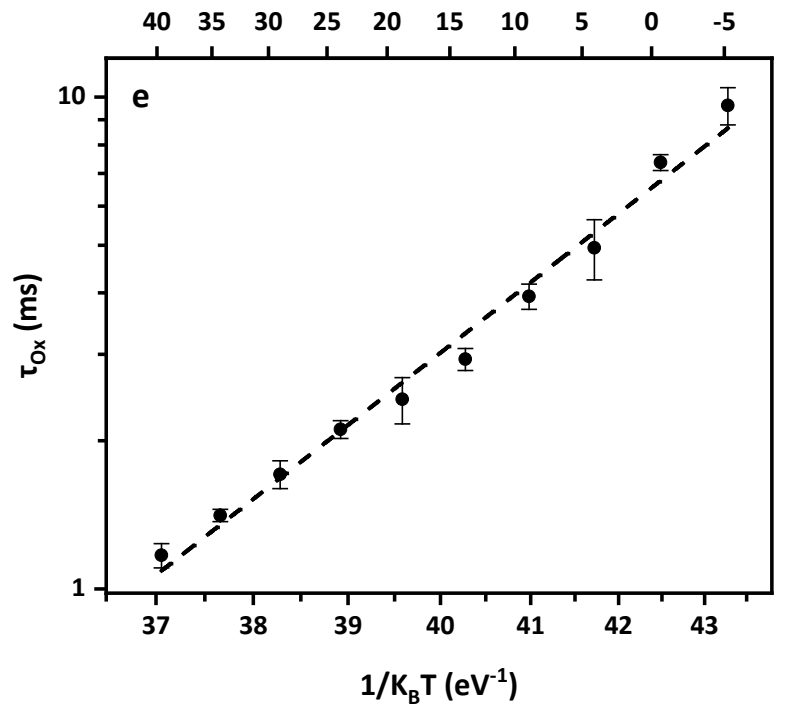

Extended Data Fig. 5: Time-resolved $\mathrm{O}_{2}$-polarography and determination of activation energy of the $\mathrm{O}_{2}$-formation step for cyanobacterial PSII from $T$. elongatus. The time courses (transients) of $\mathrm{O}_{2}$-evolution were (i) measured by time-resolved $\mathrm{O}_{2}$-polarography for a PSII layer deposited by centrifugaton on a bare platinum electrode and (ii) simulated on grounds of a physical diffusion model, including a least-square fit of the simulation parameters, as described in the Supporting information, The complete set of simulation parameters is provided in Supplementary Fig. 5. a. $\mathrm{O}_{2}$-evolution transients at temperatures ranging from $-5^{\circ} \mathrm{C}$ to $+40^{\circ} \mathrm{C}$. Each transient was obtained by averaging the averaging the all the $\mathrm{O}_{2}$-transients induced by 230 flahes of visible light. $\mathbf{b}$. The same transients as 
shown before but normalized at the respective peak value. In c and d, selected transients are shown (black symbols) with their respective fit results (coloured lines), either with original amplitudes (in c) or normalized to unity (in d). e. Arrhenius plot of $\tau_{\mathrm{ox}}$, the time constant (reciprocal rate constant) of the oxygen evolution reaction. This plot delivers an Arrhenius activation energy, $E_{\text {act, }}$ of $335+/-10$ meV with a pre-exponential frequency factor, $A$, of $2.2 \cdot 10^{8} \mathrm{~s}^{-1}$. After determination of activation energy and preexponential factor following the classical approach of Arrhenius, the enthalpy of activation $\left(H_{\text {act }}\right)$, entropy of activation ( $S_{\text {act }}$ ) were determined using the Eyring equation of transition-state theory (also called Eyring-Polanyi equation) with a transmission coefficient of unity, resulting in values of $310+/-9 \mathrm{meV}$ for $H_{\text {act }}$ and of $284+/-9 \mathrm{meV}$ for $T_{0} S_{\text {act }}\left(\right.$ with $T_{0}=20^{\circ} \mathrm{C}$ ). 


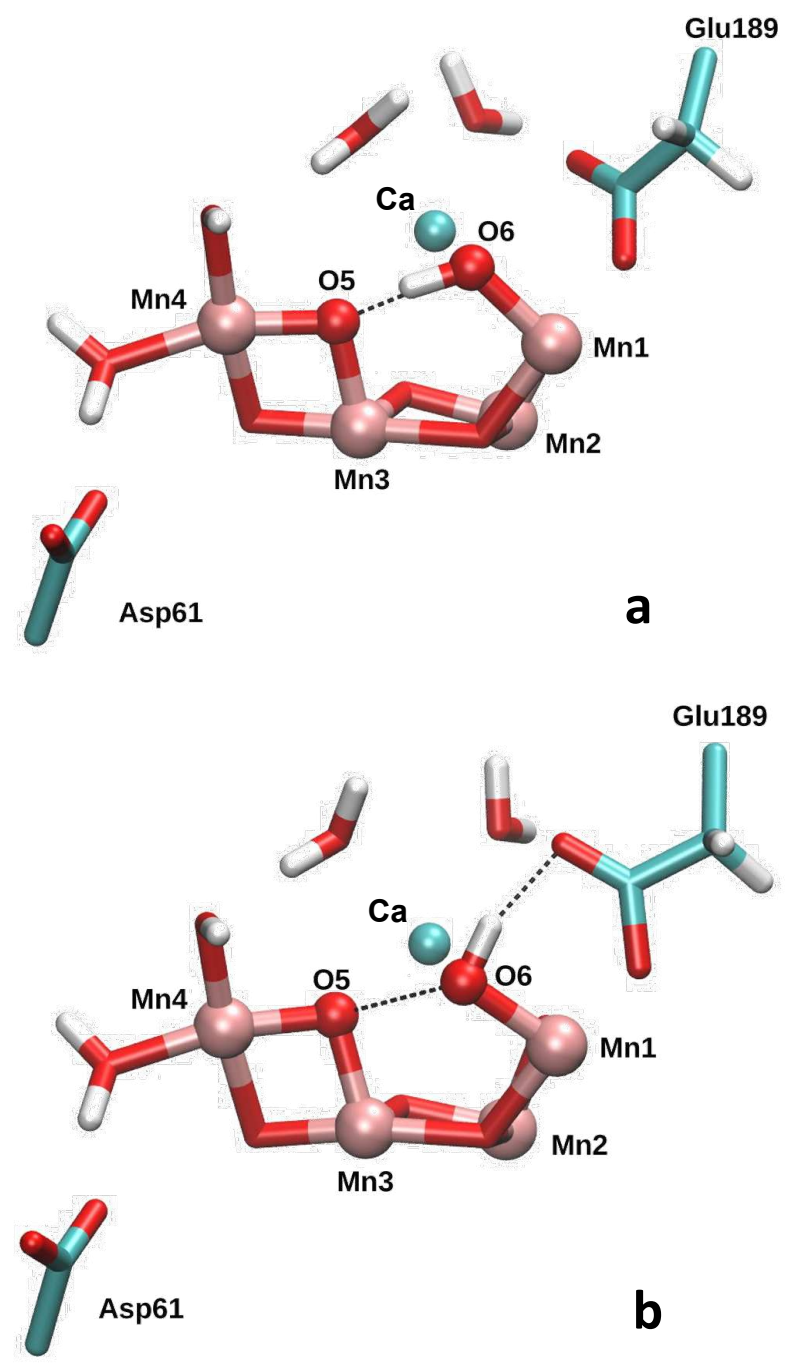

Extended Data Fig. 6: The two most stable hydrogen-bonds of protonated 06 (in both $S_{3}$ and $S_{4}$ state). Upon oxidation of TyrZ, the relative stability of the two reported conformers is reversed, conformer-a becoming more stable than conformer-b, thus favouring the oxyl radical formation as described elsewhere. ${ }^{24}$ In both cases, the deprotonated D61 is stably interacting with the W1 water molecule. Manganese atoms are shown in pink, calcium as cyan sphere, oxygen in red, carbon in cyan, and hydrogen in white. 

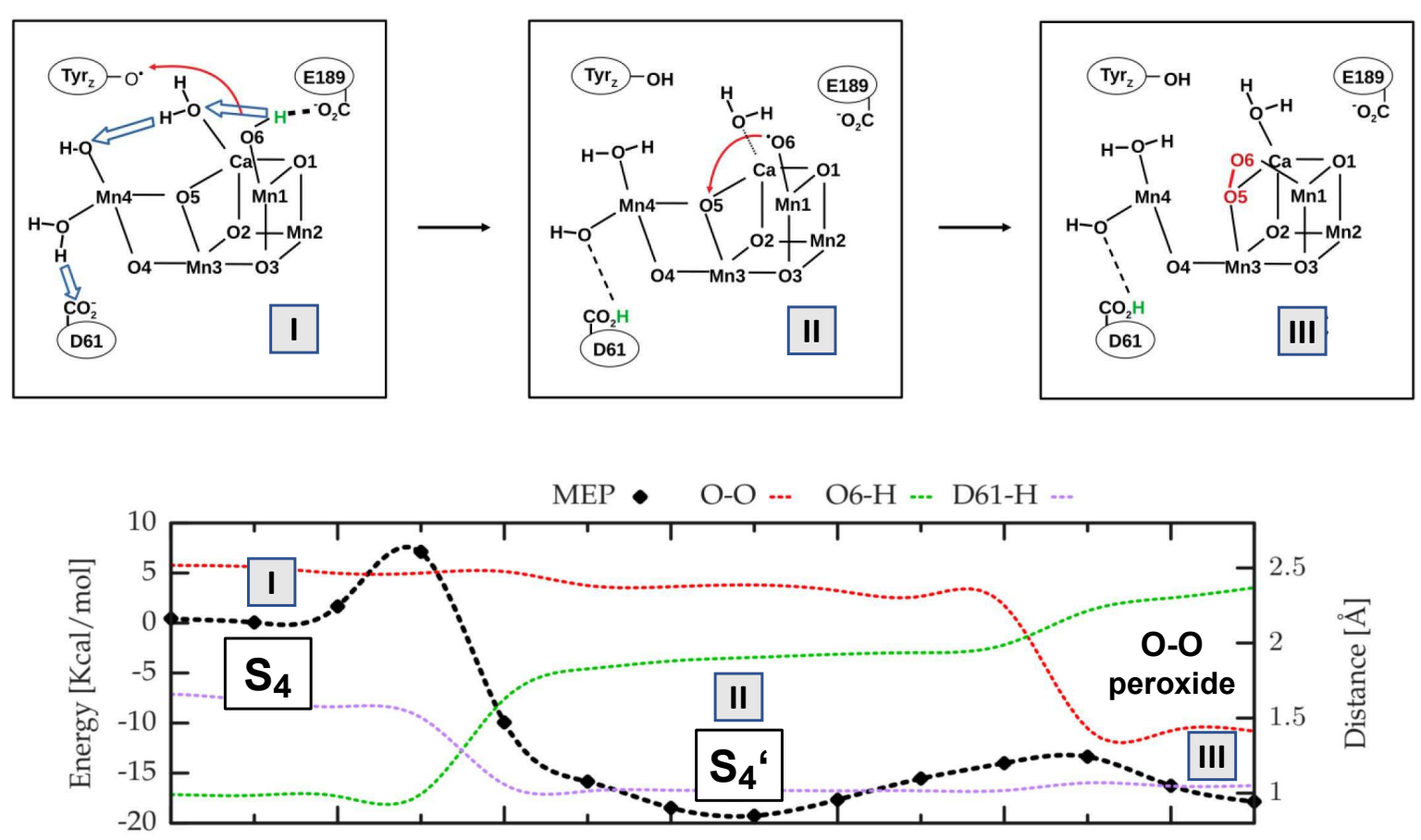

Extended Data Fig 7: Atomic and electronic rearrangements leading to peroxide formation. The top panels (I, II, and III) show the molecular sketches of the atomic and electronic motions associated with the energy barriers to overcome in the peroxide formation reaction shown in the bottom panel. 

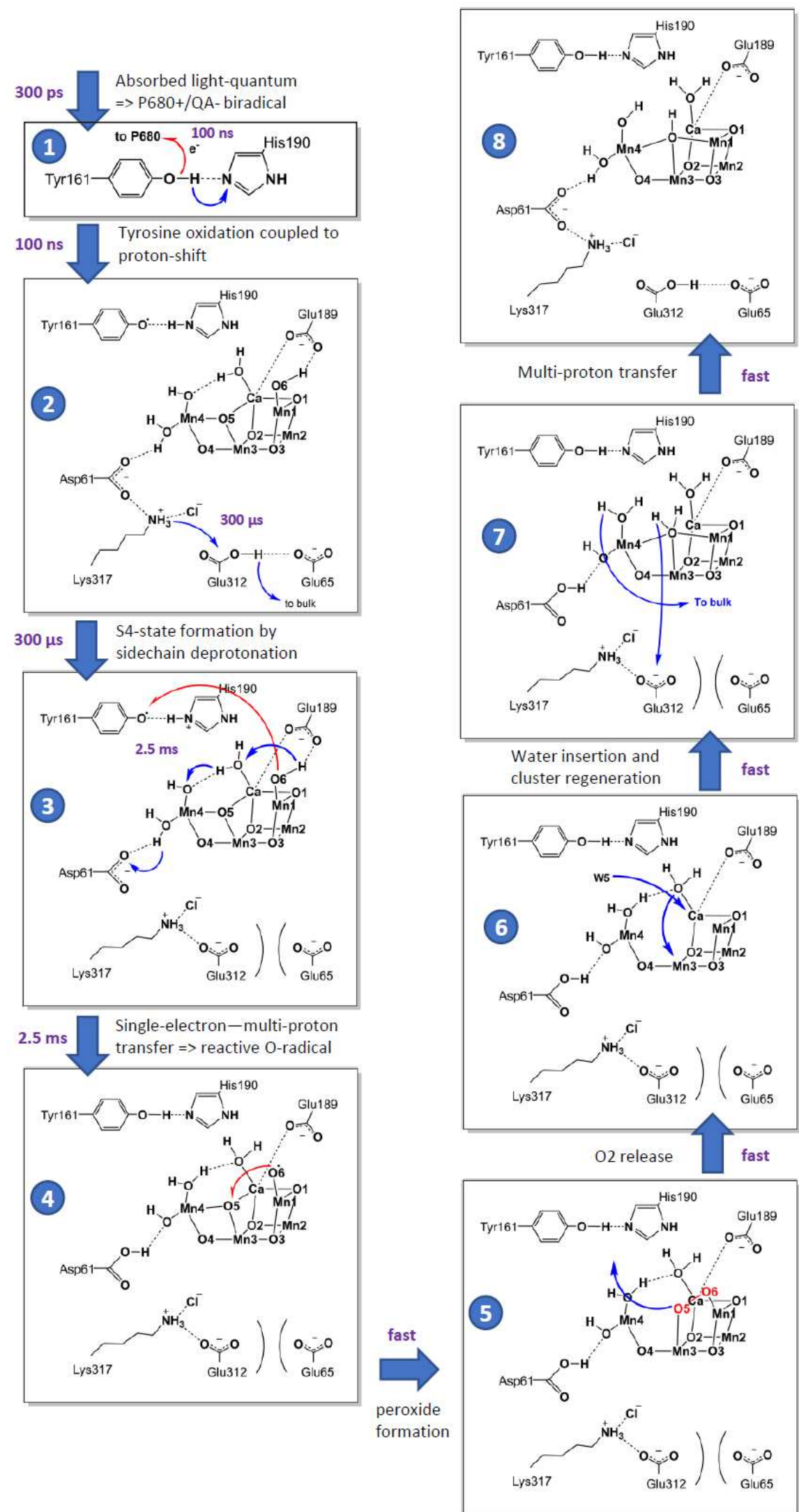

Extended Data Fig. 8: Complete picture of events in the oxygen-evolution transition as derived by computational chemistry. 

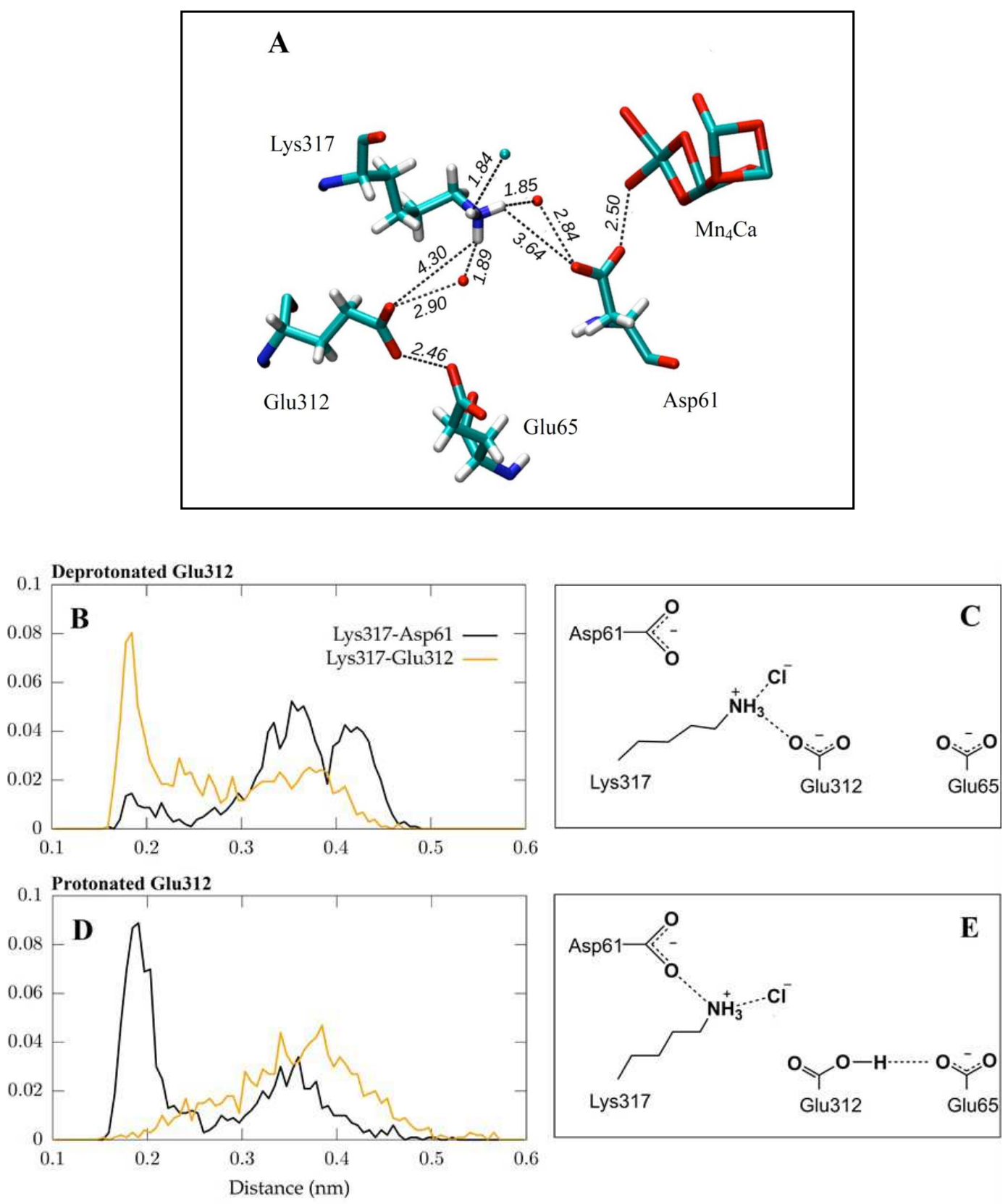

Extended Data Fig. 9: Conformations sampled by Asp61, Glu312, and Lys317. a. Sticks representation of the $\mathrm{Mn}_{4} \mathrm{Ca}$ cluster and neighbouring residues (Asp61, Glu65, Glu312 and Lys317) for the two-flash state ( $S_{3}$ state) of Photosystem II from the crystallographic model of Kern et al. (PDB ID: $6 \mathrm{DHO}$ ). $\mathrm{A} \mathrm{Cl}$ - ion as well as two water oxygens are also shown as balls. b. Distributions of distances between Lys317 and the two residues Asp61 and Glu312 sampled along 50 ns of classical MD simulation with both Asp61 and Glu312 deprotonated (simulation for $\mathrm{S}_{1}$ geometry). c. Scheme of a representative configuration of Asp61, Glu65, Glu312 and Lys317 sampled in the $S_{1}$ simulation. In this configuration Lys317 is in close contact with deprotonated Glu312. d. Distributions of distances between Lys317 and the two residues Asp61 and Glu312 sampled along 50 ns of classical MD simulation with deprotonated Asp61 and protonated Glu312 (simulation for $S_{2}$ ). e. Scheme of a representative configuration of Asp61, Glu65, Glu312 and Lys317 sampled in the $S_{2}$ simulation. Lys317 strongly interacts with Asp61, while protonated Glu312 interacts with Glu65. 


\section{References}

[currently for main manuscript text and Methods in a single list, later separated

Refs 1 to $42 \Leftrightarrow$ main manuscript text, refs. 43 to 72 for Methods section\}

1 Kok, B., Forbush, B. \& McGloin, M. Cooperation of charges in photosynthetic $\mathrm{O}_{2}$ evolution - I. A linear four-step mechanism. Photochemistry and Photobiology 11, 457-475, doi:10.1111/j.1751-1097.1970.tb06017.x (1970).

2 Junge, W. Oxygenic photosynthesis: history, status and perspective. Quarterly Reviews of Biophysics 52, ARTN e1, doi:10.1017/S0033583518000112 (2019).

3 Fischer, W. W., Hemp, J. \& Johnson, J. E. Manganese and the Evolution of Photosynthesis. Origins of Life and Evolution of Biospheres 45, 351-357, doi:10.1007/s11084-015-9442-5 (2015).

4 Cox, N., Pantazis, D. A. \& Lubitz, W. Current Understanding of the Mechanism of Water Oxidation in Photosystem II and Its Relation to XFEL Data. Annu Rev Biochem 89, 795-820, doi:10.1146/annurev-biochem-011520-104801 (2020).

5 Noguchi, T., Suzuki, H., Tsuno, M., Sugiura, M. \& Kato, C. Time-Resolved Infrared Detection of the Proton and Protein Dynamics during Photosynthetic Oxygen Evolution. Biochemistry-Us 51, 3205-3214, doi:10.1021/bi300294n (2012).

6 Shimada, Y., Kitajima-Ihara, T., Nagao, R. \& Noguchi, T. Role of the O4 Channel in Photosynthetic Water Oxidation as Revealed by Fourier Transform Infrared Difference and Time-Resolved Infrared Analysis of the D1-S169A Mutant. J Phys Chem B 124, 1470-1480, doi:10.1021/acs.jpcb.9b11946 (2020).

7 Mausle, S. M. et al. Activation energies for two steps in the S-2 -> S-3 transition of photosynthetic water oxidation from time-resolved single-frequency infrared spectroscopy. $J$ Chem Phys 153, Artn 215101, doi:10.1063/5.0027995 (2020).

8 Junge, W., Haumann, M., Ahlbrink, R., Mulkidjanian, A. \& Clausen, J. Electrostatics and proton transfer in photosynthetic water oxidation. Philosophical Transactions of the Royal Society B-Biological Sciences 357, 1407 - 1418, doi:10.1098/rstb.2002.1137 (2002).

9 Rappaport, F., Blanchard-Desce, M. \& Lavergne, J. Kinetics of electron transfer and electrochromic change during the redox transition of the photosynthetic oxygen-evolving complex. Biochimica et Biophysica Acta 1184, 178-192, doi:10.1016/0005-2728(94)90222-4 (1994).

10 Haumann, M. et al. Photosynthetic $\mathrm{O}_{2}$ formation tracked by time-resolved $\mathrm{x}$-ray experiments. Science 310, 1019-1021, doi:10.1126/science.1117551 (2005).

11 Klauss, A., Haumann, M. \& Dau, H. Alternating electron and proton transfer steps in photosynthetic water oxidation. P Natl Acad Sci USA 109, 16035-16040, doi:10.1073/pnas.1206266109 (2012). 
12 Gerencser, L. \& Dau, H. Water oxidation by Photosystem II: $\mathrm{H}_{2} \mathrm{O}-\mathrm{D}_{2} \mathrm{O}$ Exchange and the influence of $\mathrm{pH}$ support formation of an intermediate by removal of a proton before dioxygen creation. Biochemistry-Us 49, 10098-10106, doi:10.1021/bi101198n (2010).

13 Zaharieva, I., Dau, H. \& Haumann, M. Sequential and coupled proton and electron transfer events in the $S_{2} \rightarrow S_{3}$ transition of photosynthetic water oxidation revealed by time-resolved X-ray absorption spectroscopy. Biochemistry-Us 55, 6996-7004, doi:10.1021/acs.biochem.6b01078 (2016).

14 Zaharieva, I. \& Dau, H. Energetics and Kinetics of S-State Transitions Monitored by Delayed Chlorophyll Fluorescence. Frontiers in Plant Science 10, ARTN 386, doi:10.3389/fpls.2019.00386 (2019).

15 Debus, R. J. Identifying carboxylate ligand vibrational modes in photosystem II with QM/MM methods. P Natl Acad Sci USA 113, 12613-12615 (2016).

16 Nakamura, S. \& Noguchi, T. Quantum mechanics/molecular mechanics simulation of the ligand vibrations of the water-oxidizing $\mathrm{Mn} 4 \mathrm{CaO} 5$ cluster in photosystem II. P Natl Acad Sci USA 113, 12727-12732, doi:10.1073/pnas.1607897113 (2016).

17 Brahmachari, U. \& Barry, B. A. Dynamics of Proton Transfer to Internal Water during the Photosynthetic Oxygen-Evolving Cycle. J Phys Chem B 120, 11464-11473, doi:10.1021/acs.jpcb.6b10164 (2016).

18 Barth, A. Infrared spectroscopy of proteins. Bba-Bioenergetics 1767, 1073-1101, doi:10.1016/j.bbabio.2007.06.004 (2007).

19 Hussein, R. et al. Structural dynamics in the water and proton channels of photosystem II during the S2 to S3 transition. Nat Commun 12, 6531, doi:10.1038/s41467-021-26781-z (2021).

20 Wikstrom, M., Sharma, V., Kaila, V. R., Hosler, J. P. \& Hummer, G. New perspectives on proton pumping in cellular respiration. Chemical reviews 115, 2196-2221 (2015).

21 Lu, J. X. \& Gunner, M. R. Characterizing the proton loading site in cytochrome c oxidase. $P$ Natl Acad Sci USA 111, 12414-12419, doi:10.1073/pnas.1407187111 (2014).

22 Kern, J. et al. Structures of the intermediates of Kok's photosynthetic water oxidation clock. Nature 563, 421-425, doi:10.1038/s41586-018-0681-2 (2018).

23 Suga, M. et al. Light-induced structural changes and the site of $\mathrm{O}=\mathrm{O}$ bond formation in PSII caught by XFEL. Nature 543, 131-135, doi:10.1038/nature21400 (2017).

24 Narzi, D., Capone, M., Bovi, D. \& Guidoni, L. Evolution from $\mathrm{S}_{3}$ to $\mathrm{S}_{4}$ state of the oxygen evolving complex in Photosystem II monitored by QM/MM dynamics. Chemistry--A European Journal (2018).

25 Siegbahn, P. E. M. Nucleophilic water attack is not a possible mechanism for O-O bond formation in photosystem II. P Natl Acad Sci USA 114, 4966-4968, doi:10.1073/pnas.1617843114 (2017). 
26 Capone, M., Guidoni, L. \& Narzi, D. Structural and dynamical characterization of the S-4 state of the Kok-Joliot's cycle by means of QM/MM Molecular Dynamics Simulations. Chem Phys Lett 742, ARTN 137111, doi:10.1016/j.cplett.2020.137111 (2020).

27 Guerra, F., Siemers, M., Mielack, C. \& Bondar, A. N. Dynamics of Long-Distance HydrogenBond Networks in Photosystem II. Journal of Physical Chemistry B 122, 4625-4641, doi:10.1021/acs.jpcb.8b00649 (2018).

$28 \mathrm{Li}, \mathrm{H}$. J. et al. Capturing structural changes of the S-1 to S-2 transition of photosystem II using time-resolved serial femtosecond crystallography. lucrj 8, 431-443, doi:10.1107/S2052252521002177 (2021).

29 Narzi, D., Bovi, D. \& Guidoni, L. Pathway for Mn-cluster oxidation by tyrosine-Z in the S-2 state of photosystem II. P Natl Acad Sci USA 111, 8723-8728, doi:10.1073/pnas.1401719111 (2014).

30 Shoji, M. et al. Large-scale QM/MM calculations of the CaMn4O5 cluster in the S3 state of the oxygen evolving complex of photosystem II. Comparison between water-inserted and no water-inserted structures. Faraday Discuss 198, 83-106, doi:10.1039/c6fd00230g (2017).

31 Retegan, M. et al. A five-coordinate Mn(IV) intermediate in biological water oxidation: spectroscopic signature and a pivot mechanism for water binding. Chemical Science 7, 72-84, doi:10.1039/c5sc03124a (2016).

32 Wang, J., Askerka, M., Brudvig, G. W. \& Batista, V. S. Crystallographic Data Support the Carousel Mechanism of Water Supply to the Oxygen-Evolving Complex of Photosystem II. ACS Energy Lett 2, 2299-2306, doi:10.1021/acsenergylett.7b00750 (2017).

33 Amin, M. et al. Proton-Coupled Electron Transfer During the S-State Transitions of the Oxygen-Evolving Complex of Photosystem II. Journal of Physical Chemistry B 119, 7366-7377, doi:10.1021/jp510948e (2015).

34 Kuroda, H. et al. Proton transfer pathway from the oxygen-evolving complex in photosystem II substantiated by extensive mutagenesis. Biochim Biophys Acta Bioenerg 1862, 148329, doi:10.1016/j.bbabio.2020.148329 (2021).

35 Siegbahn, P. E. A structure-consistent mechanism for dioxygen formation in photosystem II. Chemistry--A European Journal 14, 8290-8302 (2008).

36 Siegbahn, P. E. Mechanisms for proton release during water oxidation in the S2 to S3 and S3 to S4 transitions in photosystem II. Phys Chem Chem Phys 14, 4849-4856, doi:10.1039/c2cp00034b (2012).

37 Suga, M. et al. An oxyl/oxo mechanism for oxygen-oxygen coupling in PSIl revealed by an $x$ ray free-electron laser. Science 366, 334-+ (2019).

38 de Lichtenberg, C. \& Messinger, J. Substrate water exchange in the S2 state of photosystem II is dependent on the conformation of the Mn4Ca cluster. Phys Chem Chem Phys 22, 1289412908, doi:10.1039/d0cp01380c (2020).

39 Ugur, I., Rutherford, A. W. \& Kaila, V. R. I. Redox-coupled substrate water reorganization in the active site of Photosystem II-The role of calcium in substrate water delivery. BbaBioenergetics 1857, 740-748, doi:10.1016/j.bbabio.2016.01.015 (2016). 
Saito, K., Nakagawa, M. \& Ishikita, H. pK(a)of the ligand water molecules in the oxygenevolving $\mathrm{Mn}(4) \mathrm{CaO}(5)$ cluster in photosystem II. Commun Chem 3, ARTN 89, doi:10.1038/s42004-020-00336-7 (2020).

41 Risch, M. et al. Water oxidation by amorphous cobalt-based oxides: in situ tracking of redox transitions and mode of catalysis. Energy \& Environmental Science 8, 661-674, doi:Doi 10.1039/C4ee03004d (2015).

42 Gorlin, M. et al. Oxygen Evolution Reaction Dynamics, Faradaic Charge Efficiency, and the Active Metal Redox States of Ni-Fe Oxide Water Splitting Electrocatalysts. J Am Chem Soc 138, 5603-5614, doi:10.1021/jacs.6b00332 (2016).

43 Nong, H. N. et al. Key role of chemistry versus bias in electrocatalytic oxygen evolution. Nature 587, 408-413, doi:10.1038/s41586-020-2908-2 (2020).

44 Zaharieva, I. et al. Water oxidation catalysis - role of redox and structural dynamics in biological photosynthesis and inorganic manganese oxides. Energy Environ. Sci. 9, 2433-2443, doi:10.1039/C6EE01222A (2016).

45 Schiller, H. \& Dau, H. Preparation protocols for high-activity photosystem II membrane particles of green algae and higher plants, $\mathrm{pH}$ dependence of oxygen evolution and comparison of the $\mathrm{S}_{2}$-state multiline signal by X-band EPR spectroscopy. Journal of Photochemistry and Photobiology B: Biology 55, 138-144, doi:10.1016/S10111344(00)00036-1 (2000).

46 Iuzzolino, L., Dittmer, J., Dörner, W., Meyer-Klaucke, W. \& Dau, H. X-ray absorption spectroscopy on layered photosystem II membrane particles suggests manganese-centered oxidation of the oxygen-evolving complex for the $\mathrm{S}_{0}-\mathrm{S}_{1}, \mathrm{~S}_{1}-\mathrm{S}_{2}$, and $\mathrm{S}_{2}-\mathrm{S}_{3}$ transitions of the water oxidation cycle. Biochemistry-Us 37, 17112-17119 (1998).

47 Styring, S., Sjöholm, J. \& Mamedov, F. Two tyrosines that changed the world: Interfacing the oxidizing power of photochemistry to water splitting in photosystem II. Biochimica et Biophysica Acta 1817, 76-87, doi:10.1016/j.bbabio.2011.03.016 (2012).

48 Schuth, N. et al. Inhibitory and non-inhibitory $\mathrm{NH}_{3}$ binding at the water-oxidizing manganese complex of photosystem II suggests possible sites and a rearrangement mode of substrate water molecules. Biochemistry-Us 56, 6240-6256, doi:10.1021/acs.biochem.7b00743 (2017).

49 Dilbeck, P. L. et al. The mutation D1-D61N in Synechocystis sp. PCC 6803 allows the observation of $\mathrm{pH}$-sensitive intermediates in the formation and release of $\mathrm{O}_{2}$ from Photosystem II. Biochemistry-Us 51, 1079-1091, doi:10.1021/bi201659f (2012).

50 Guo, Y. et al. Unusual kinetics of thermal decay of dim-light photoreceptors in vertebrate vision. Proceedings of the National Academy of Sciences 111, 10438-10443 (2014).

51 Bao, H. \& Burnap, R. L. Structural rearrangements preceding dioxygen formation by the water oxidation complex of photosystem II. P Natl Acad Sci USA 112, E6139-E6147, doi:10.1073/pnas.1512008112 (2015).

52 Hornak, V. et al. Comparison of multiple amber force fields and development of improved protein backbone parameters. Proteins 65, 712-725, doi:10.1002/prot.21123 (2006). 
53 Wang, J. M., Wolf, R. M., Caldwell, J. W., Kollman, P. A. \& Case, D. A. Development and testing of a general amber force field (vol 25, pg 1157, 2004). J Comput Chem 26, 114-114, doi:10.1002/jcc.20145 (2005).

54 Laino, T., Mohamed, F., Laio, A. \& Parrinello, M. An efficient real space multigrid OM/MM electrostatic coupling. J Chem Theory Comput 1, 1176-1184, doi:10.1021/ct050123f (2005).

55 Nose, S. A Unified Formulation of the Constant Temperature Molecular-Dynamics Methods. J Chem Phys 81, 511-519, doi:Doi 10.1063/1.447334 (1984).

56 Hoover, W. G. Canonical Dynamics - Equilibrium Phase-Space Distributions. Phys Rev A 31, 1695-1697, doi:DOI 10.1103/PhysRevA.31.1695 (1985).

57 Bovi, D., Narzi, D. \& Guidoni, L. Magnetic interactions in the catalyst used by nature to split water: a DFT plus U multiscale study on the $\mathrm{Mn} 4 \mathrm{CaO} 5$ core in photosystem II. New Journal of Physics 16, Artn 015020, doi:10.1088/1367-2630/16/1/015020 (2014).

58 VandeVondele, J. et al. QUICKSTEP: Fast and accurate density functional calculations using a mixed Gaussian and plane waves approach. Comput Phys Commun 167, 103-128, doi:10.1016/j.cpc.2004.12.014 (2005).

59 Becke, A. D. A New Mixing of Hartree-Fock and Local Density-Functional Theories. J Chem Phys 98, 1372-1377, doi:Doi 10.1063/1.464304 (1993).

60 Lee, C. T., Yang, W. T. \& Parr, R. G. Development of the Colle-Salvetti Correlation-Energy Formula into a Functional of the Electron-Density. Phys Rev B 37, 785-789, doi:DOI 10.1103/PhysRevB.37.785 (1988).

61 Capone, M., Bovi, D., Narzi, D. \& Guidoni, L. Reorganization of Substrate Waters between the Closed and Open Cubane Conformers during the S-2 to S-3 Transition in the Oxygen Evolving Complex. Biochemistry-Us 54, 6439-6442, doi:10.1021/acs.biochem.5b00782 (2015).

62 Capone, M., Narzi, D., Bovi, D. \& Guidoni, L. Mechanism of Water Delivery to the Active Site of Photosystem II along the S-2 to S-3 Transition. Journal of Physical Chemistry Letters 7, 592596, doi:10.1021/acs.jpclett.5b02851 (2016).

63 Shoji, M., Isobe, H., Shigeta, Y., Nakajima, T. \& Yamaguchi, K. Concerted Mechanism of Water Insertion and $\mathrm{O} 2$ Release during the S4 to SO Transition of the Oxygen-Evolving Complex in Photosystem II. J Phys Chem B 122, 6491-6502, doi:10.1021/acs.jpcb.8b03465 (2018).

64 Krewald, V. et al. Metal oxidation states in biological water splitting. Chemical Science 6, 1676-1695, doi:10.1039/C4SC03720K (2015).

65 Cox, N., Pantazis, D. A., Neese, F. \& Lubitz, W. Biological water oxidation. Accounts of Chemical Research 46, 1588-1596, doi:Doi 10.1021/Ar3003249 (2013).

66 Hutter, J., lannuzzi, M., Schiffmann, F. \& VandeVondele, J. CP2K: atomistic simulations of condensed matter systems. Wires Comput Mol Sci 4, 15-25, doi:10.1002/wcms.1159 (2014).

67 Henkelman, G. \& Jonsson, H. Improved tangent estimate in the nudged elastic band method for finding minimum energy paths and saddle points. J Chem Phys 113, 9978-9985, doi:10.1063/1.1323224 (2000). 
68 Narzi, D., Mattioli, G., Bovi, D. \& Guidoni, L. A Spotlight on the Compatibility between XFEL and Ab Initio Structures of the Oxygen Evolving Complex in Photosystem II. Chem-Eur J 23, 6969-6973, doi:10.1002/chem.201700722 (2017).

69 Siu, S. W., Vacha, R., Jungwirth, P. \& Bockmann, R. A. Biomolecular simulations of membranes: physical properties from different force fields. J Chem Phys 128, 125103, doi:10.1063/1.2897760 (2008).

70 Jorgensen, W. L., Chandrasekhar, J., Madura, J. D., Impey, R. W. \& Klein, M. L. Comparison of Simple Potential Functions for Simulating Liquid Water. J Chem Phys 79, 926-935, doi:Doi 10.1063/1.445869 (1983).

71 Van der Spoel, D. et al. GROMACS: Fast, flexible, and free. J Comput Chem 26, 1701-1718, doi:10.1002/jcc.20291 (2005).

72 Darden, T., York, D. \& Pedersen, L. Particle Mesh Ewald - an N.Log(N) Method for Ewald Sums in Large Systems. J Chem Phys 98, 10089-10092, doi:Doi 10.1063/1.464397 (1993).

73 Hess, B., Bekker, H., Berendsen, H. J. C. \& Fraaije, J. G. E. M. LINCS: A linear constraint solver for molecular simulations. J Comput Chem 18, 1463-1472, doi:Doi 10.1002/(Sici)1096987x(199709)18:12<1463::Aid-Jcc4>3.3.Co;2-L (1997).

74 Nose, S. \& Klein, M. L. Constant Pressure Molecular-Dynamics for Molecular-Systems. Mol Phys 50, 1055-1076, doi:Doi 10.1080/00268978300102851 (1983).

75 Parrinello, M. \& Rahman, A. Polymorphic Transitions in Single-Crystals - a New MolecularDynamics Method. J Appl Phys 52, 7182-7190, doi:Doi 10.1063/1.328693 (1981).

76 Süss, B. Entwicklung eines Step-Scan FTIR Experiments zur Untersuchung der lichtinduzierten Wasserspaltung der oxygenen Photosynthese Dr. rer. nat. thesis, Freie Universität Berlin, (2011).

77 Görlin, M. Light-induced and time-resolved FTIR difference spectroscopy of photosystem II Master thesis, Uppsala University, (2012). 


\section{Supplementary Files}

This is a list of supplementary files associated with this preprint. Click to download.

- PhotosyntheticO2GreifeetalSI.pdf

- Peroxobondformation.mp4 